\title{
An integrated map of structural variation in 2,504 human genomes
}

A list of authors and their affiliations appears at the end of the paper.

Structural variants are implicated in numerous diseases and make up the majority of varying nucleotides among human genomes. Here we describe an integrated set of eight structural variant classes comprising both balanced and unbalanced variants, which we constructed using short-read DNA sequencing data and statistically phased onto haplotype blocks in 26 human populations. Analysing this set, we identify numerous gene-intersecting structural variants exhibiting population stratification and describe naturally occurring homozygous gene knockouts that suggest the dispensability of a variety of human genes. We demonstrate that structural variants are enriched on haplotypes identified by genome-wide association studies and exhibit enrichment for expression quantitative trait loci. Additionally, we uncover appreciable levels of structural variant complexity at different scales, including genic loci subject to clusters of repeated rearrangement and complex structural variants with multiple breakpoints likely to have formed through individual mutational events. Our catalogue will enhance future studies into structural variant demography, functional impact and disease association.

Structural variants (SVs), including deletions, insertions, duplications and inversions, account for most varying base pairs (bp) among individual human genomes ${ }^{1}$. Numerous studies have implicated SVs in human health with associated phenotypes ranging from cognitive disabilities to predispositions to obesity, cancer and other maladies $^{1,2}$. Discovery and genotyping of these variants remains challenging, however, since SVs are prone to arise in repetitive regions and internal SV structures can be complex ${ }^{3}$. This has created challenges for genome-wide association studies (GWAS) ${ }^{4,5}$. Despite recent methodological and technological advances ${ }^{6-9}$, efforts to perform discovery, genotyping, and statistical haplotype-block integration of all major SV classes have so far been lacking. Earlier SV surveys depended on microarrays ${ }^{10}$ as well as genomic and clone-based approaches limited to a small number of samples ${ }^{11-15}$. More recently, short-read DNA sequencing data from the initial phases of the 1000 Genomes Project $^{8,9}$ enabled us to construct sets of SVs, genotyped across populations, with enhanced size and breakpoint resolution ${ }^{6,7}$. Previous 1000 Genomes Project SV set releases, however, encompassed fewer individuals and were largely ${ }^{6}$ or entirely ${ }^{8}$ limited to deletions, in spite of the relevance of other SV classes to human genetics ${ }^{1,2,4}$.

The objective of the Structural Variation Analysis Group has been to discover and genotype major classes of SVs (defined as DNA variants $\geq 50 \mathrm{bp}$ ) in diverse populations and to generate a statistically phased reference panel with these SVs. Here we report an integrated map of 68,818 SVs in unrelated individuals with ancestry from 26 populations (Supplementary Table 1). We constructed this resource by analysing 1000 Genomes Project phase 3 whole-genome sequencing (WGS) data ${ }^{16}$ along with data from orthogonal techniques, including long-read single-molecule sequencing (Supplementary Table 2), to characterize hitherto unresolved SV classes. Our study emphasizes the population diversity of SVs, quantifies their functional impact, and highlights previously understudied SV classes, including inversions exhibiting marked sequence complexity.

\section{Construction of our phase $3 \mathrm{SV}$ release}

We mapped Illumina WGS data $(\sim 100$ bp reads, mean 7.4 -fold coverage) from 2,504 individuals onto an amended version ${ }^{8}$ of the GRCh37 reference assembly using two independent mapping
algorithms-BWA ${ }^{17}$ and mrsFAST ${ }^{18}$ - and performed SV discovery and genotyping using an ensemble of nine different algorithms (Extended Data Fig. 1 and Supplementary Note). We applied several orthogonal experimental platforms for SV set assessment, refinement and characterization (Supplementary Table 2) and to calculate the false discovery rate (FDR) for each SV class (Table 1). Callset refinements facilitated through long-read sequencing enabled us to incorporate a number of additional SVs into our callset, including an additional 698 inversions and 9,132 small ( $<1 \mathrm{kbp})$ deletions, compared to the SV set released with the 1000 Genomes Project marker paper $^{16}$. As a result, our callset differs slightly relative to the marker paper's SV set ${ }^{16}$ (see Supplementary Table 2). We merged individual callsets to construct our unified release (Table 1), comprising 42,279 biallelic deletions, 6,025 biallelic duplications, 2,929 mCNVs (multi allelic copy-number variants), 786 inversions, 168 nuclear mitochondrial insertions (NUMTs), and 16,631 mobile element insertions (MEIs, including 12,748, 3,048 and 835 insertions of Alu, L1 and SVA (SINE-R, VNTR and Alu composite) elements, respectively).

$\mathrm{SV}$ non-reference genotype concordance estimates ranged from $\sim 98 \%$ for biallelic deletions and MEI classes to $\sim 94 \%$ for biallelic duplications. $60 \%$ of SVs were novel with respect to the Database of Genomic Variants (DGV) ${ }^{19}(50 \%$ reciprocal overlap criterion, Fig. 1a), whereby $71 \%$ of SVs (50\% reciprocal overlap) and $60 \%$ of collapsed copy-number variable regions (CNVRs, 1 bp overlap) were novel compared to previous 1000 Genomes Project releases ${ }^{6,8}$, reflecting methodological improvements and inclusion of additional populations. Novel SVs showed enrichment for rare sites, which we detected down to an autosomal allele count of ' 1 '. And while variations in FDR estimates were evident with SV size and VAF (variant allele frequency), we consistently estimated the FDR at $\leq 5.4 \%$ when stratifying deletions and duplications by size and frequency, including for rare SVs with VAF $<0.1 \%$ (Extended Data Figs 1, 2). A comparison with deep-coverage Complete Genomics (CG) sequencing data indicated an overall sensitivity of $88 \%$ for deletions and $65 \%$ for duplications, with the false negatives driven largely by the relatively lowered sensitivity for ascertaining small SVs in Illumina sequencing data (Fig. 1b, Extended Data Fig. 3). The average per-individual sensitivity was similar for deletions (89\%) and slightly lower for 
Table 1 | Phase 3 extended SV release

\begin{tabular}{|c|c|c|c|c|c|c|c|c|}
\hline SV class & No. sites & $\begin{array}{l}\text { Median size of SV } \\
\text { sites (bp) }\end{array}$ & $\begin{array}{l}\text { Median kbp } \\
\text { per individual }\end{array}$ & $\begin{array}{l}\text { Median alleles } \\
\text { per individual }\end{array}$ & Site FDR & $\begin{array}{l}\text { Biallelic site breakpoint } \\
\text { precision (bp) }\end{array}$ & $\begin{array}{l}\text { Genotype concordance } \\
\text { (non-ref.) }\end{array}$ & $\begin{array}{l}\text { Sensitivity } \\
\text { estimates }\end{array}$ \\
\hline Deletion (biallelic) & 42,279 & 2,455 & 5,615 & 2,788 & $2 \% *-4 \% \dagger$ & $15( \pm 50) * * 0.7( \pm 9.5) \dagger \dagger$ & $98 \%$ & $88 \%$ \\
\hline Duplication (biallelic) & 6,025 & 35,890 & 518 & 17 & $1 \% *-4 \% \dagger$ & $683( \pm 1,350)+t$ & $94 \%$ & $65 \%$ \\
\hline $\mathrm{mCNV}$ & 2,929 & 19,466 & 11,346 & 340 & $1 \% *-4 \% \dagger$ & - & NA & NA \\
\hline Inversion & 786 & 1,697 & 78 & 37 & $17 \% \S(9 \%)+||||$ & $32( \pm 47)||||$ & $96 \% \S$ & $32 \%$ \\
\hline $\mathrm{MEI}$ & 16,631 & 297 & 691 & 1,218 & $4 \%$ & $0.95( \pm 5.93)$ & $98 \% \|$ & $83 \#-96 \% \star$ \\
\hline NUMT & 168 & 157 & 3 & 5.3 & $10 \% *$ & $0.25( \pm 0.43)$ & $86.1 \%$ & NA \\
\hline
\end{tabular}

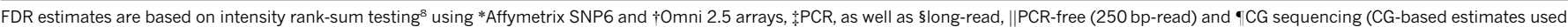
reciprocal overlaps of $50 \%$ and $20 \%$ for deletions and duplications, respectively). Estimate by comparing MEls to all \#calls or all $\star P C R$-validated calls from ${ }^{20}$ (estimates for individual MEl classes are in

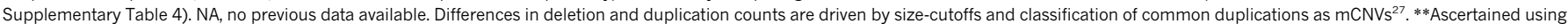
read-pairs or read-depth. $\dot{\dagger} \dagger$ Ascertained with split-reads ${ }^{23}$. + Estimated for tandem duplications. ||||Estimated for inversions with paired-end support from both breakpoints.

duplications (50\%). For MEI classes, estimated sensitivities ranged from $83-96 \%$ (Table 1) compared to the 1000 Genomes Project pilot phase where a different MEI detection tool was used ${ }^{20}$. For inversions, we estimated an overall sensitivity of $32 \%$ based on variants with a positive validation status recorded in the InvFEST database ${ }^{21}$, with an increased sensitivity of $67 \%$ for inversions $<5 \mathrm{kbp}$ in size.

We performed breakpoint assembly using pooled Illumina WGS and Pacific Biosciences (PacBio) sequencing data ${ }^{22}$, and additionally performed split-read analysis ${ }^{23}$ of short reads, to resolve the fineresolution breakpoint structure of 37,250 SVs (29,954 deletions, 357 tandem duplications, 6,919 MEIs, and 20 inversions; Supplementary Table 3). Breakpoint assemblies showed a mean boundary precision of $0-15 \mathrm{bp}$ for all SV types, with the exception of inversions and duplications for which we achieved mean precision estimates of $32 \mathrm{bp}$ and $683 \mathrm{bp}$, respectively (Table 1, Fig. 1c).

\section{Population genetic properties of SVs}

We explored the population genetic properties of SVs among five continental groups-Africa (AFR), the Americas (AMR), East Asia (EAS), Europe (EUR) and South Asia (SAS). The bulk of SVs occur at low frequency $(65 \%$ exhibit VAF $<0.2 \%)$ consistent amongst individual SV classes (Extended Data Figs 2,3). While rare SVs are typically specific to individual continental groups, at VAF $\geq 2 \%$ nearly all SVs are shared across continents (Fig. 1d, Extended Data Fig. 3). Notably, we identified 1,075 SVs with VAF $>50 \%$ (889 biallelic deletions, 2 biallelic duplications, 90 mCNVs, 88 MEIs and 6 inversions) encompassing $5 \mathrm{Mbp}$, sites of interest for future updates to the human reference genome. We estimated the mutation rate for each SV class using Waterson's estimator of $\theta$, for example, ascertaining a mutation rate of 0.113 deletions per haploid genome generation, a threefold higher estimate compared with previous reports ${ }^{10,24}$, probably owing to our increased power for detecting variants $<5 \mathrm{kbp}$ (Supplementary Note).

We found that $73 \%$ of SVs with $>1 \%$ VAF and $68 \%$ of rarer SVs (VAF $>0.1 \%$ ) are in linkage disequilibrium (LD) with nearby single nucleotide polymorphisms (SNPs) $\left(r^{2}>0.6\right)$; however, the proportion of variants in LD highly depends on the SV class (Fig. 1e, Extended Data Fig. 4). For example, only $44 \%$ of all biallelic duplications with VAF $>0.1 \%$ were in LD with a nearby SNP $\left(r^{2}>0.6\right)$, in agreement with previous findings ${ }^{10,25,26}$. Notably, we observed a striking depletion of biallelic duplications amongst common SVs $\left(P<2 \times 10^{-16}\right.$, Kolmogorov-Smirnov test; Extended Data Fig. 5) with most common duplications classified as multi-allelic SVs (that is, $\mathrm{mCNVs}$ ). This behaviour suggests extensive recurrence of SVs at duplication sites consistent with what was recently observed in a smaller cohort of 849 individuals ${ }^{27}$. These LD characteristics suggest duplications are currently under-ascertained for disease associations using tag-SNP-based approaches.

Based on our haplotype-resolved SV catalogue, we observed that individuals of African ancestry exhibit, on average, $27 \%$ more heterozygous deletions than individuals from other populations (mean of 1,705 versus 1,342), consistent with $\mathrm{SNPs}^{28}$ (Extended Data Fig. 5). The relative proportion of deletion- versus SNP-affected sequence, however, showed a $13 \%$ excess in non-African compared to African populations (ratio 1.64 versus 1.45). Principal component analyses with different SV classes generally recapitulated continental population structure and admixture (Extended Data Fig. 6 and Supplementary Note). Our analysis further allowed us to identify a catalogue of 6,495 ancestry-informative MEI markers of potential value to population genetics history and forensics research (Extended Data Fig. 5, Supplementary Table 4). $\mathbf{a}$
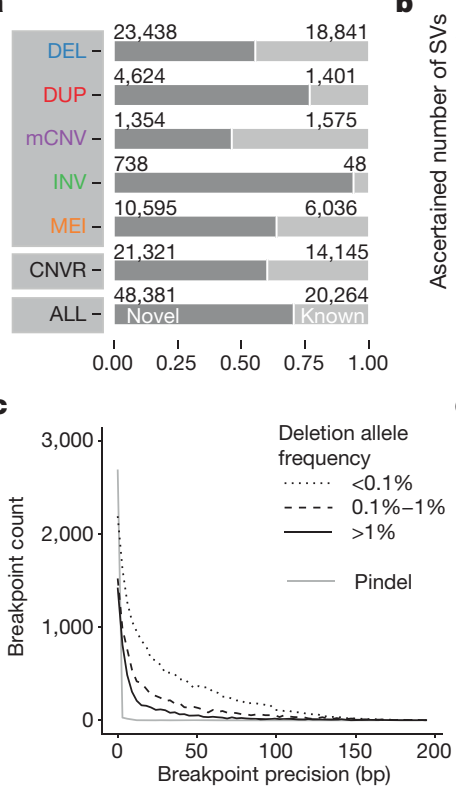

b
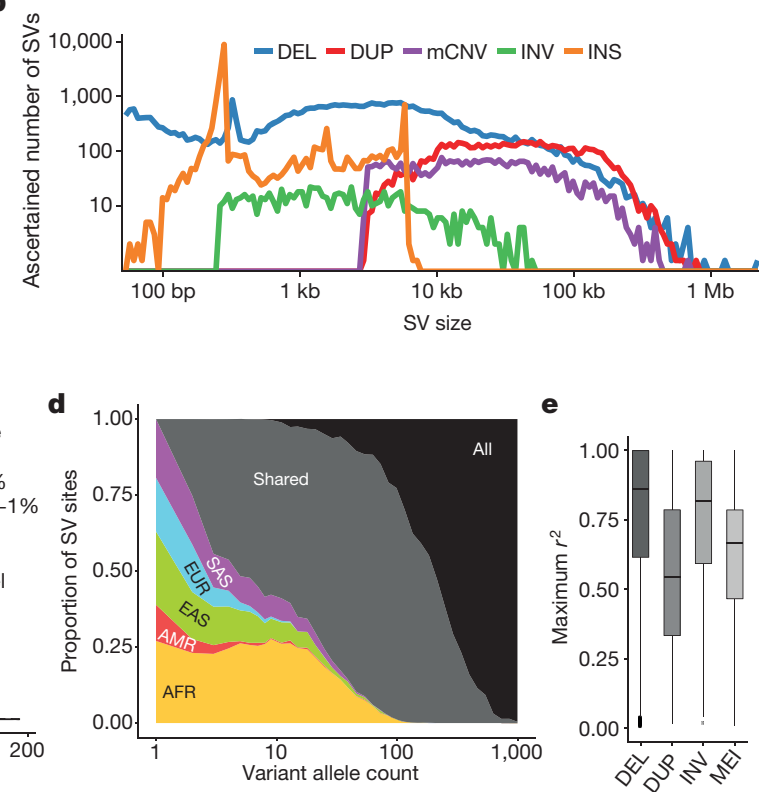

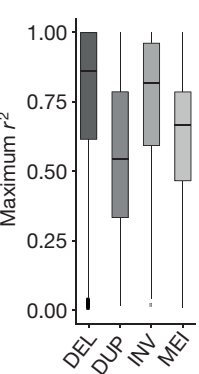

Figure $1 \mid$ Phase 3 integrated SV callset. a, Novelty based on overlap of our SV set with $\mathrm{DGV}^{19}$ (upper panel, broken down by SV class), of collapsed CNVRs with earlier 1000 Genomes Project releases $^{6,8}$ (middle panel) and of our SV set with refs 6, 8 (bottom panel). b, Size distribution of ascertained SVs (bin width is uniform in log-scale). DEL, biallelic deletion, DUP, biallelic duplication, INV, inversion, INS, non-reference insertion (including MEIs and NUMTs). c, Breakpoint precision of assembled deletions stratified by VAF (split-read caller Pindel ${ }^{23}$ shown separately). d, SV allele sharing across continental groups. e, LD properties of biallelic SV classes. 
Since population stratification can be used as a signature to detect adaptive selection, we additionally identified SVs varying in VAF amongst different populations. For each SV site we calculated a $V_{\mathrm{ST}}$ statistic, a measure highly correlated with $F_{\mathrm{ST}}$ (the fixation index ${ }^{29}$ that can be applied to assess population stratification of biallelic and multi-allelic SVs ${ }^{29}$. We observed 1,434 highly stratified SVs ( $>0.2 V_{\mathrm{ST}}$, corresponding to 2.9 standard deviations (s.d.) from the mean; Supplementary Table 5), among which 578 intersected gene coding sequences (CDSs). Among these were several SVs associated with regions previously reported to be under positive selection, such as KANSL1 mCNVs (Extended Data Fig. 6) that tag a Europeanenriched inversion polymorphism associated with increased fecundity $^{30}$. Most of the population-stratified sites, however, have not been previously described and are, thus, potential targets for future investigation of SVs undergoing adaptive selection or genetic drift. These include, for example, a $14.5 \mathrm{kbp}$ intronic duplication of HERC2 enriched in East Asians ( $V_{\mathrm{ST}}=0.62$ EAS-EUR).

\section{Functional impact of SVs}

We analysed the intersection of deletions binned by VAF with various classes of genic and intergenic functional elements (Fig. 2a, Extended Data Fig. 7). The CDSs, untranslated regions (UTRs) and introns of genes, in addition to $\mathrm{ENCODE}^{31}$ transcription factor binding sites and ultrasensitive noncoding regions, showed a significant depletion $(P<0.001$; permutation testing in each VAF bin) compared to a random background model. In general, these elements are more depleted (in terms of fold change) in common VAF bins compared to rarer deletion alleles, in keeping with purifying ${ }^{10}$ (or in some cases background $^{32}$ ) selection. Genes more intolerant to mutation (as measured from SNP diversity, residual variation intolerance score $\left.(\text { RVIS })^{33}<20\right)$ exhibited the most pronounced depletion $(P<0.001$ permutation testing between pairs of RVIS-score categories). All other SV classes exhibited similar signatures of selection; when compared to deletions these depletions were, however, more attenuated (Fig. 2b, Extended Data Fig. 7). Additional assessment of the site frequency spectrum showed that, as deletion sizes increase, these SV s become rarer $\left(P<2.2 \times 10^{-16}\right.$; linear model, F-test $)$, evidence of purifying selection against events more likely intersecting functional elements. Duplications, by comparison, did not exhibit such trend, consistent with reduced selective constraints (Supplementary Note).

We additionally analysed 5,819 homozygous deletions to search for gene knockouts occurring naturally in human populations. Among these we identified 240 genes (corresponding to 204 individual deletion sites) that, on the basis of the observation of homozygous losses in normal individuals, seem to be 'dispensable' (Supplementary Table 6). Most of the underlying deletions were found in more than one human population, and for only one $(0.5 \%)$ we observed evidence for the putative involvement of uniparental disomy in the homozygosity (Supplementary Note). The majority $(>80 \%)$ of these homozygous gene losses were novel compared to a previous analysis based on DGV variants ${ }^{19}$, or recent clinical genomics studies (Supplementary Note). As expected, genes affected by homozygous loss were not highly conserved and were relatively tolerant to other forms of genetic variation (RVIS $=0.74$ compared to OMIM disease genes showing RVIS $=0.43$; $P=9.4 \times 10^{-25}$; Mann-Whitney test). Moreover, the set was functionally enriched for glycoproteins (Benjamini-Hochberg corrected $P$-value $=1.6 \times 10^{-3}$, EASE (Expression Analysis Systematic Explorer) score) and genes harbouring immunoglobulin domains (BenjaminiHochberg corrected $P$-value $=1.0 \times 10^{-5}$, EASE score).

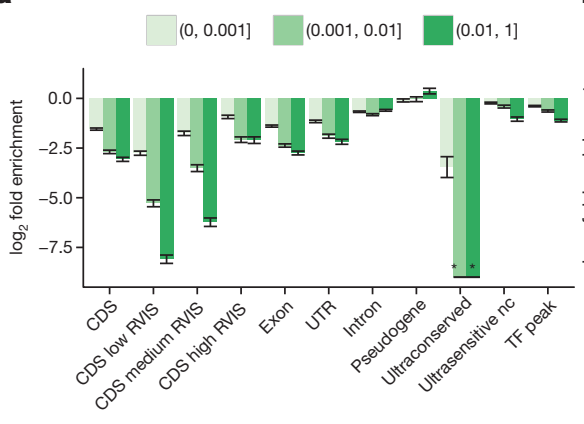

c
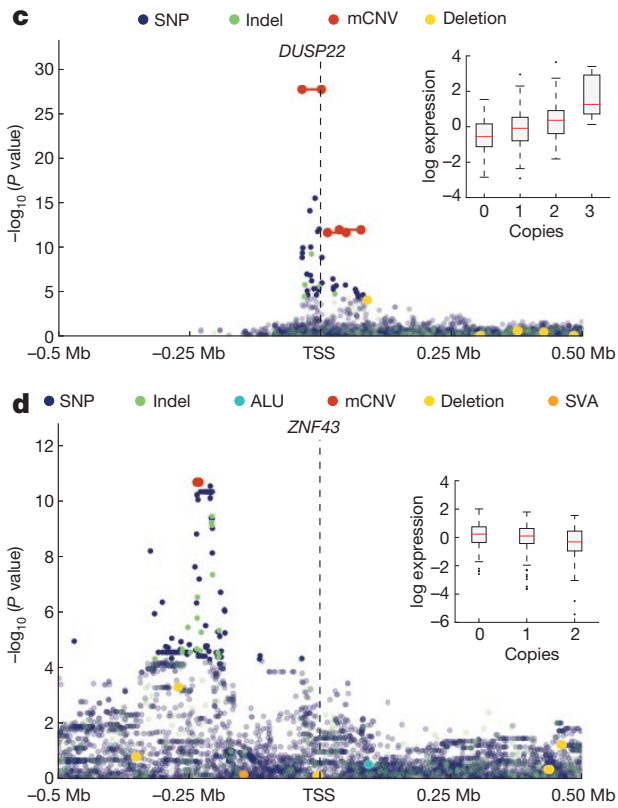

DEL DUP INV MCNV

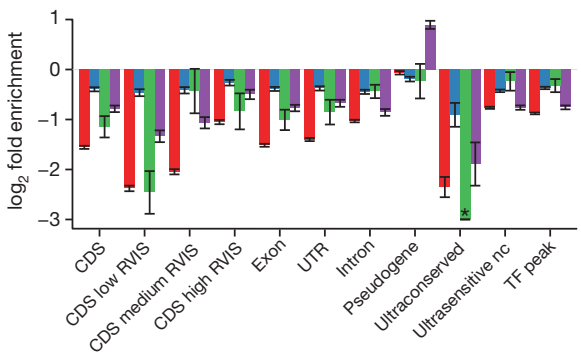

e

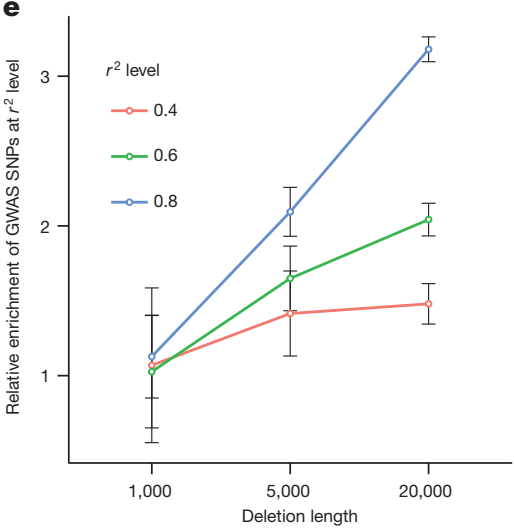

Figure $2 \mid$ SV functional impact. a, Relative enrichment or depletion of genomic elements within breakpoint-resolved deletions binned by VAF. TF, transcription factor binding site; nc, noncoding. RVIS range from $0-100$ (low $<20$, medium $20-50$, high $\geq 50$ ). *no element intersected. b, Enrichment/depletion of genomic elements within different SV classes, compared with breakpoint-resolved deletions. c, Manhattan plot of DUSP22-eQTL. Inset, boxplots of association between copy-number genotype and expression. d, Manhattan plot of ZNF43-eQTL. e, Enrichment of SV-containing haplotypes at previously reported GWAS hits (error bars show s.e.m.). 
We next quantified the functional impact of SVs using expression quantitative trait loci (eQTL) associations as a surrogate ${ }^{34,35}$. Based on transcriptome data from lymphoblastoid cell lines derived from 462 individuals $^{36}$ (the gEUVADIS consortium), we tested 18,969 expressed protein-coding genes for cis-eQTL associations, considering $1 \mathrm{Mbp}$ candidate regions upstream and downstream of CDSs. A joint eQTL analysis using SNPs, indels and SVs with VAF > 1\% identified 54 eQTLs with a lead SV association (denoted SV-eQTL) and 9,537 eQTLs with a lead SNP/indel association (10\% FDR). For an additional 166 eQTLs with lead associations to SNPs or indels, we observed SVs in LD $\left(r^{2}>0.5\right)$ seven times more than when using random variants matched for LD structure, distance to the transcription start site, and VAF, suggesting that a larger number of eQTLs are probably affected by SVs (Extended Data Fig. 8, Supplementary Table 7). In proportion to the number of variants tested, SV classes were up to $\sim 50$-fold enriched for SV-eQTLs $\left(P=2.84 \times 10^{-39}\right.$, one-sided Fisher's exact test; Supplementary Table 8). Large SVs were associated with increased effect size; for example, a twofold increase in effect size for genic SVs $>10 \mathrm{kbp}$ versus variants $<1 \mathrm{kbp}(P=0.0004 ; t$-test; Extended Data Fig. 8). Taken together, although SNPs contribute more eQTLs overall, our results suggest that SVs have a disproportionate impact on gene expression relative to their number.

Among those 220 eQTLs having either an SV-eQTL or an SV in $\mathrm{LD}$ with the lead SNP/indel, most were due to deletions (55\% of associations), followed by mCNVs (19\%) (Supplementary Table 8). Although SV-eQTLs with the largest effect sizes tended to overlap with CDSs, such as for the dual specificity phosphatase 22 (DUSP22) gene (Fig. 2c), we also observed several expression-associated SVs strictly intersecting upstream noncoding sequences, including an mCNV upstream of ZNF43 (Fig. 2d) possibly mediated through variation of a cis-regulatory element. We additionally considered the impact of accounting for SVs when constructing personalized reference genomes for transcriptome analysis. To illustrate this, we considered RNA read alignments for the sample NA12878, comparing the standard reference genome with GRCh37-derived personalized references constructed using NA12878 SNPs, or using NA12878 SNPs and SVs. Using such an approach, we observed marked changes in expression for 525 exons ( \pm 10 reads, $\geq$ onefold change relative to the standard reference), 24 of which could be attributed to the inclusion of SVs into the personalized reference (Supplementary Table 9).

The relevance of SVs to eQTLs suggests that a number of disease associations previously detected by GWAS may be attributable to SVs, which are difficult to assess directly in GWAS. To test this hypothesis we compared 12,892 previously reported SNP-based GWAS hits to SVs identified in our data set, identifying 136 candidate SVs in strong LD $\left(r^{2}>0.8\right)$ with GWAS variants, which represents a 1.5 -fold enrichment when compared to a VAF and haplotype size-matched background set and a threefold enrichment for deletions $>20 \mathrm{kbp}$ $(P=0.004)$ (Fig. 2e and Supplementary Note). Approximately a third of these candidate GWAS associations (39) were novel, impacting phenotypes such as colorectal cancer and bone mineral density (Supplementary Table 10). Interestingly, 64\% of these novel associations were mediated by deletions $<1 \mathrm{kbp}$, a size range for which our study has improved power over previous surveys, which more than doubles (from 18 to 40 ) the number of SVs $<1 \mathrm{kbp}$ in strong LD with a GWAS lead SNP. Thus, our SV resource could facilitate discovery of numerous additional disease-linked SVs.

\section{SV clustering and complexity}

Advances in Illumina sequencing towards longer read lengths $(\sim 100 \text { bp versus } 36 \mathrm{bp})^{6}$ in conjunction with the population-level data allowed us to perform an in-depth investigation of SV complexity and clustering. We identified 3,163 regions where SVs seemed to cluster ( $>2$ SVs mapping within 500 bp; Supplementary Table 11). To reduce redundancy caused by multiple overlapping calls per sample, we cal- culated distinct CNVRs per cluster by merging calls per sample and haplotype and then counting the distinct CNVRs produced across samples (average 6.4 \pm 7.2 CNVRs per cluster). We identified 30 genomic regions with an excess of CNVRs ( $>4$ s.d. or $>36 \mathrm{CNVRs}$ per cluster). This clustering effect was not correlated with segmental duplications $(\mathrm{r}=0.02)$ and only partially explained by SNP diversity $(r=0.15$; Extended Data Fig. 9). CNVR clusters showed enrichment near late-replicating origins $(P=0.013$, permutation test $)$ and at cytogenically defined 'fragile' sites $(P=0.0017$; permutation test). Although the proportion of gene content in regions exhibiting excessive SV clustering was significantly reduced when compared to a null distribution $(P<0.000001$, permutation test), 1,881 of 3,163 such regions (59\%) intersected one or more genes (Supplementary Table 11). This includes a region comprised of 47 SVs (ranking 2nd out of the 30 genomic regions with $>4$ s.d.) encompassing the pregnancy-specific glycoprotein gene family (Fig. 3a), a set of genes thought to be critically important for maintenance of pregnancy ${ }^{37}$. Other SV clusters associated with genes (for example, IMMP2L, CHL1 and GRID2) have been implicated as potential risk factors for disease, including neurodevelopmental disorders ${ }^{38}$.

We additionally specifically assessed the complexity of the 29,954 deletions with resolved breakpoints and found that $6 \%(1,822)$ intersected another deletion with distinct breakpoints. A larger fraction ( $16 \%$ or 4,813 of assembled deletion sites) showed the presence of additional inserted sequence at deletion breakpoints. We grouped 1,651 deletions with mean size of $3.1 \mathrm{kbp}$ and at least $10 \mathrm{bp}$ of additional DNA sequence between the original SV site boundaries into five broad classes (Fig. 3b, Supplementary Table 12). The most common class $(n=501,30.3 \%)$, termed 'Ins with Dup and Del', comprised deletions exhibiting a recognizable duplicated sequence interval within the respective inserted sequence. Notably, in many cases $(n=191)$ the inserted sequences comprised two or more apparent sequence duplications at the deletion boundaries (a class denoted 'Ins with MultiDup and Del'). Additional classes commonly observed include Inv and Del (inversion with adjacent deletion; $n=9$ ) and 'MultiDel'-a class where two or more adjacent deletions are separated by at least one sequence 'spacer' of up to $\sim 204$ bp in length $(n=370)$. However, not all complex SVs fit into these classes, with 214 sites forming distinct patterns corresponding to multiple classes or exhibiting increased complexity. Template-switching mechanisms could explain the notable complexity of these $\mathrm{SVs}^{3}$. Indeed, microhomology patterns were typically present between the breakpoints of deletions and the respective boundaries of insertion templates at these sites (Extended Data Fig. 9), consistent with formation through single mutational events (Supplementary Note). Across the complex sites assessed, $871(53 \%)$ showed evidence for a local template ( $\geq 10 \mathrm{bp}$ match, within $10 \mathrm{kbp}$ ), whereas for 41 the insertion was presumably templated from a distal region ( $\geq 22 \mathrm{bp}$ match, $>10 \mathrm{kbp}$ away), including 17 sites where the DNA stretch was likely derived from RNA templates (Supplementary Table 13).

To further characterize SV breakpoint complexity, we employed two alternative approaches that do not rely on low-coverage Illumina read assembly. We first examined 7,804 small deletions for breakpoint complexity using split-read analysis ${ }^{23}$ (Fig. 3c) and identified 664 (median size $67 \mathrm{bp}$ ) exhibiting complexity, 64 of which contained insertions $\geq 3 \mathrm{bp}$ that may be derived from a nearby template (Supplementary Table 14, Extended Data Fig. 9). We additionally realigned long DNA reads from a single individual (NA12878) ${ }^{22}$ sequenced by high-coverage PacBio (median read length $3.0 \mathrm{kbp}$ ) and Moleculo (median $3.2 \mathrm{kbp}$ ) single-molecule WGS around deletions from our release set (Fig. 3d). Out of 766 deletions in NA12878 investigated with this approach, 62 exhibited complexity showing three to six breakpoints (Supplementary Table 12). A deletion of exon 3 of the serine protease inhibitor SPINK14, for example, was accompanied by an inversion of an internal segment of the SV sequence (Fig. 3d, left panel). In contrast to the smaller proportion 


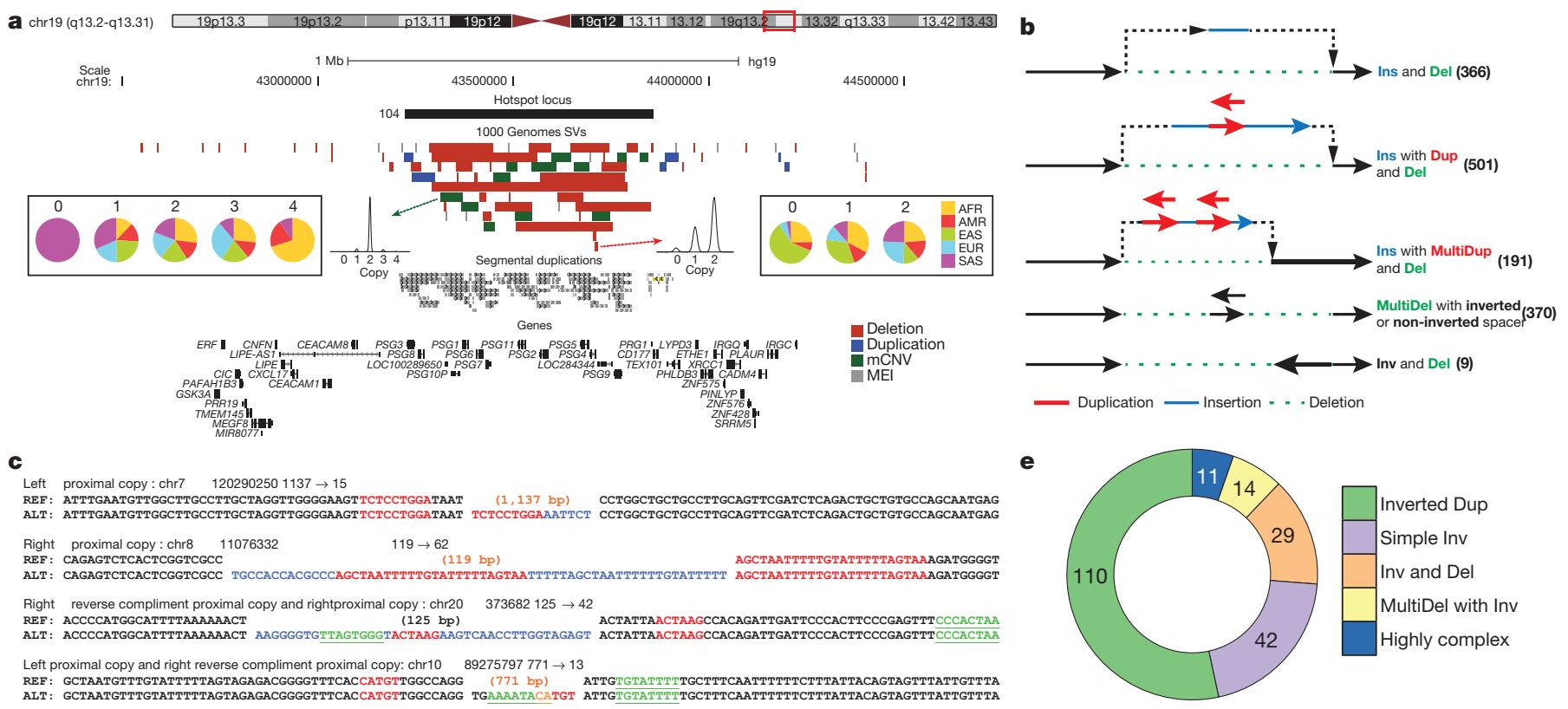

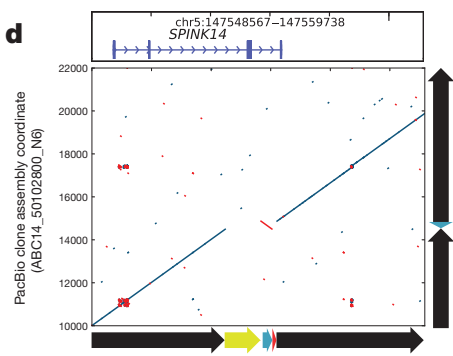

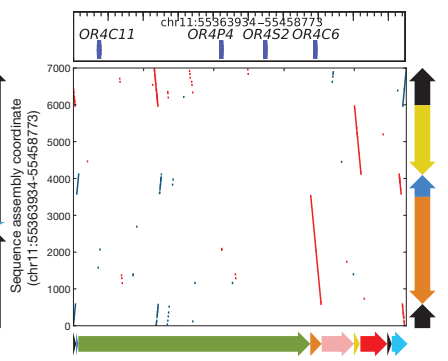

Figure $3 \mid$ SV complexity at different scales. a, PSG locus with clustered SVs. Population copy-number state histograms are shown for two example SVs. b, Schemes depicting assembled complex deletions. c, Smaller-scale complex deletions identified with Pindel $^{23}$. Flanking sequences are shown for reference (REF) and alternate (ALT) alleles, further to insertions at the breakpoints.

of deletions showing breakpoint complexity, the majority of inversions assessed in NA12878 (19/28) exhibited multiple breakpoints.

To further explore inversion sequence complexity, we performed a battery of targeted analyses, leveraging PacBio resequencing of fosmids (targeting 34 loci), sequencing by Oxford Nanopore Minion (60 loci) and PacBio (206 loci) of long-range PCR amplicons, and data for 13 loci from another sample (CHM1) sequenced by highcoverage PacBio $\mathrm{WGS}^{14}$. Altogether we verified and further characterized 229 inversion sites, 208 using long-read data and 21 by PCR (Supplementary Table 15), increasing the number of known validated inversions ${ }^{21}$ by $>2.5$-fold. Remarkably, only $20 \%$ of all sequenced inversions characterized in this manner were 'simple' (termed 'Simple Inv'), exhibiting two breakpoints (Fig. 3e), including a $2 \mathrm{kbp}$ inversion on chromosome 4 intersecting a regulatory exon of the Ras homologue family member $\mathrm{RHOH}$ (Fig. 3d, right panel). The majority of inversions (54\%) corresponded to inverted duplications ('Inverted Dup'; Fig. 3d, middle right panel). In nearly all cases, these involved duplicated stretches $<1 \mathrm{kbp}$ inserted within $5 \mathrm{kbp}$ of the alternate copy, suggesting a common mechanism of SV formation (Extended Data Fig. 10). The remaining inversions comprised 'Inv and Del' events (14\%), 'MultiDel' events exhibiting inverted spacers (7\%), and more highly complex sites (5\%; Fig. $3 \mathrm{~d}$, middle left panel). The appreciable inversion complexity uncovered here is most likely due to a mutational process forming complex SVs, potentially involving DNA replication errors ${ }^{3}$, rather than due to recurrent rearrangement, as our analyses failed to detect corresponding intermediate events in 1000 Genomes Project samples.

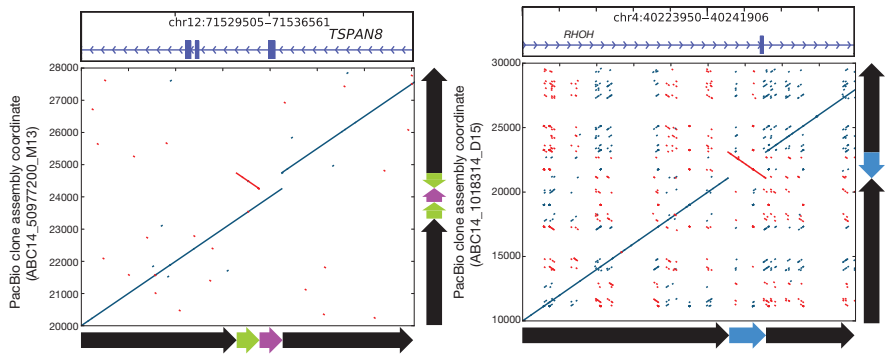

Proximal stretches matching the insertion are labelled in red (forward) and green (reverse complement). Blue, insertions lacking nearby matches. d, Alignment dot plots depicting inversions (inverted sequences are in red within each dot plot). Adjacent schemes depict allelic structures for REF and ALT. e, Inversion complexity summarized.

\section{Discussion}

We present what is to our knowledge the most comprehensive set of human SVs to date as an integrated resource for future disease and population genetics studies. We estimate that individuals harbour a median of $18.4 \mathrm{Mbp}$ of SVs per diploid genome, an excess contributed to a large extent by mCNVs (11.3 Mbp) and biallelic deletions (5.6 Mbp; Table 1). When collapsing mCNV sites carrying multiple copies as well as homozygous SVs onto the haploid reference assembly, a median of $8.9 \mathrm{Mbp}$ of sequence are affected by SVs, compared to $3.6 \mathrm{Mbp}$ for SNPs. Furthermore, 37,250 SVs have mapped breakpoints amounting to $>113 \mathrm{Mbp}$ of $\mathrm{SV}$ sequence resolved at the nucleotide-level. By mining homozygous deletions we identified over two hundred nonessential human genes, a set enriched for immunoglobulin domains that hence may reflect variation in the immune repertoire underlying inter-individual differences in disease susceptibility.

We demonstrate that SV classes are disproportionally enriched (by up to $\sim 50$-fold) for SV-eQTLs, although only 220 SVs were found either as lead eQTL association or in high LD with the respective lead SNP. While this corresponds to proportionally fewer associations relative to SNPs compared to a prior estimate based on array techno$\log y^{34}$, this may be explained by the reliance of this prior estimate on bacterial artificial chromosome arrays, which ascertain large SVs $(>50 \mathrm{kbp})$ that associate with strong effect size, as well as by the relative scarcity of SNPs tested in an earlier study ${ }^{34}$ (HapMap Phase I $)^{39}$. We further expand the number of candidate SVs in strong LD with GWAS hits by 30\% (39/136 novel associations implicating SVs as candidates) and find that GWAS haplotypes are enriched up to 
threefold for common SVs, which emphasizes the relevance of ascertaining SVs in disease studies. The large number of novel SVs smaller than $1 \mathrm{kbp}$ in length associated with previously reported GWAS hits highlights the importance of increasing sensitivity for SV detection and genotyping at this size range. Additionally, the large number of rare SVs captured by our resource may be of value for disease association studies investigating rare variants.

Our deep population survey has identified hotspots of SV mutation that cannot be accounted for by deep coalescence or segmental duplication content. We describe hitherto undescribed patterns of SV complexity, particularly for inversions. These patterns indicate that other more complex mutational processes outside of non-allelic homologous recombination, retrotransposition, and non-homologous end-joining played an important role in shaping our genome. In spite of this, it remains difficult to fully disentangle the contributions of SV mutation rates and selective forces to the observed variant clustering. The findings presented here leveraged substantial recent technological advances, including increases in Illumina read length and developments in long-read DNA technologies. SV discovery remains a challenge nonetheless, and the full complexity and spectrum of SV is not yet understood. Our analyses, for example, are largely based on 7.4-fold Illumina WGS and, thus, are underpowered to capture much of the complexity of variation, including SVs in repetitive regions, non-reference insertions, and short SVs at the boundaries of the detection limits of read-depth and paired-end-based SV discovery ${ }^{4}$ Furthermore, while many SVs in our callset are statistically phased, the diploid nature of the genome is non-optimally captured by current analysis approaches, which mostly rely on mapping to a haploid reference. We envision that in the future, the use of technology allowing substantial increases in read lengths over the current stateof-the-art will enable genomic analyses of truly diploid sequences to facilitate targeting these additional layers of genomic complexity. Until this is realized, our SV set represents an invaluable resource for the construction and analysis of personalized genomes.

Online Content Methods, along with any additional Extended Data display items and Source Data, are available in the online version of the paper; references unique to these sections appear only in the online paper.

\section{Received 19 February; accepted 20 August 2015}

1. Weischenfeldt, J., Symmons, O., Spitz, F. \& Korbel, J. O. Phenotypic impact of genomic structural variation: insights from and for human disease. Nature Rev. Genet 14, 125-138 (2013)

2. Malhotra, D. \& Sebat, J. CNVs: harbingers of a rare variant revolution in psychiatric genetics. Cell 148, 1223-1241 (2012)

3. Hastings, P. J., Lupski, J. R., Rosenberg, S. M. \& Ira, G. Mechanisms of change in gene copy number. Nature Rev. Genet. 10, 551-564 (2009).

4. Alkan, C., Coe, B. P. \& Eichler, E. E. Genome structural variation discovery and genotyping. Nature Rev. Genet. 12, 363-376 (2011).

5. Wellcome Trust Case Control Consortium. Genome-wide association study of CNVs in 16,000 cases of eight common diseases and 3,000 shared controls. Nature 464, 713-720 (2010)

6. Mills, R. E. et al. Mapping copy number variation by population-scale genome sequencing. Nature 470, 59-65 (2011).

7. Sudmant, P. H. et al. Diversity of human copy number variation and multicopy genes. Science 330, 641-646 (2010).

8. The 1000 Genomes Project Consortium. An integrated map of genetic variation from 1,092 human genomes. Nature 491, 56-65 (2012).

9. The 1000 Genomes Project Consortium. A map of human genome variation from population-scale sequencing. Nature 467, 1061-1073 (2010).

10. Conrad, D. F. et al. Origins and functional impact of copy number variation in the human genome. Nature 464, 704-712 (2010).

11. Kidd, J. M. et al. A human genome structural variation sequencing resource reveals insights into mutational mechanisms. Cell 143, 837-847 (2010).

12. Korbel, J. O. et al. Paired-end mapping reveals extensive structural variation in the human genome. Science 318, 420-426 (2007).

13. Pang, A. W. et al. Towards a comprehensive structural variation map of an individual human genome. Genome Biol. 11, R52 (2010).

14. Chaisson, M. J. et al. Resolving the complexity of the human genome using singlemolecule sequencing. Nature 517, 608-611 (2015).

15. Teague, B. et al. High-resolution human genome structure by single-molecule analysis. Proc. Natl Acad. Sci. USA 107, 10848-10853 (2010)

16. The 1000 Genomes Project Consortium. A global reference for human genetic variation. Nature http://dx.doi.org/10.1038/nature15393 (this issue).
17. Li, H. \& Durbin, R. Fast and accurate long-read alignment with Burrows-Wheeler transform. Bioinformatics 26, 589-595 (2010).

18. Hach, F. et al. mrsFAST-Ultra: a compact, SNP-aware mapper for high performance sequencing applications. Nucleic Acids Res. 42, W494-W500 (2014).

19. MacDonald, J. R., Ziman, R., Yuen, R. K., Feuk, L. \& Scherer, S. W. The Database of Genomic Variants: a curated collection of structural variation in the human genome. Nucleic Acids Res. 42, D986-D992 (2014).

20. Stewart, C. et al. A comprehensive map of mobile element insertion polymorphisms in humans. PLoS Genet. 7, e1002236 (2011).

21. Martínez-Fundichely, A. et al. InvFEST, a database integrating information of polymorphic inversions in the human genome. Nucleic Acids Res. 42 , D1027-D1032 (2014)

22. Pendleton, M. et al. Assembly and diploid architecture of an individual human genome via single-molecule technologies. Nature Methods 12, 780-786 (2015).

23. Ye, K., Schulz, M. H., Long, Q., Apweiler, R. \& Ning, Z. Pindel: a pattern growth approach to detect break points of large deletions and medium sized insertions from paired-end short reads. Bioinformatics 25, 2865-2871 (2009)

24. Kloosterman, W. P. et al. Characteristics of de novo structural changes in the human genome. Genome Res. 25, 792-801 (2015).

25. McCarroll, S. A. et al. Integrated detection and population-genetic analysis of SNPS and copy number variation. Nature Genet. 40, 1166-1174 (2008).

26. Locke, D. P. et al. Linkage disequilibrium and heritability of copy-number polymorphisms within duplicated regions of the human genome. Am. J. Hum Genet. 79, 275-290 (2006).

27. Handsaker, R. E. et al. Large multiallelic copy number variations in humans. Nature Genet 47, 296-303 (2015)

28. Simons, Y. B., Turchin, M. C., Pritchard, J. K. \& Sella, G. The deleterious mutation load is insensitive to recent population history. Nature Genet. 46, 220-224 (2014)

29. Redon, R. etal. Global variation in copy number in the human genome. Nature $\mathbf{4 4 4}$ 444-454 (2006)

30. Stefansson, H. et al. A common inversion under selection in Europeans. Nature Genet. 37, 129-137 (2005).

31. ENCODE Project Consortium. An integrated encyclopedia of DNA elements in the human genome. Nature 489, 57-74 (2012).

32. McVicker, G., Gordon, D., Davis, C. \& Green, P. Widespread genomic signatures of natural selection in hominid evolution. PLOS Genet. 5, e1000471 (2009).

33. Petrovski, S., Wang, Q., Heinzen, E. L., Allen, A. S. \& Goldstein, D. B. Genic intolerance to functional variation and the interpretation of personal genomes. PLoS Genet. 9, e1003709 (2013)

34. Stranger, B. E. et al. Relative impact of nucleotide and copy number variation on gene expression phenotypes. Science 315, 848-853 (2007)

35. Schlattl, A., Anders, S., Waszak, S. M., Huber, W. \& Korbel, J. O. Relating CNVs to transcriptome data at fine resolution: assessment of the effect of variant size, type, and overlap with functional regions. Genome Res. 21, 2004-2013 (2011).

36. Lappalainen, T. et al. Transcriptome and genome sequencing uncovers functional variation in humans. Nature 501, 506-511 (2013).

37. Moore, T. \& Dveksler, G. S. Pregnancy-specific glycoproteins: complex gene families regulating maternal-fetal interactions. Int. J. Dev. Biol. 58, 273-280 (2014).

38. Girirajan, S. et al. Relative burden of large CNVs on a range of neurodevelopmental phenotypes. PLoS Genet. 7, e1002334 (2011)

39. International HapMap Consortium. A haplotype map of the human genome. Nature 437, 1299-1320 (2005).

40. Conrad, D. F. et al. Mutation spectrum revealed by breakpoint sequencing of human germline CNVs. Nature Genet. 42, 385-391 (2010).

Supplementary Information is available in the online version of the paper

Acknowledgements We thank M. Hurles, R. Durbin and D. Reich for valuable comments during the preparation of this work, S. Scherer for providing PCR-based inversion genotyping data for the initial calibration of our inversion caller, B. Nelson and V. Benes for technical assistance, and T. Brown and N. Habermann for critical review of the manuscript. The following people are acknowledged for contributing to PacBio sequencing or analysis: E. Patel, S. Lee, H. Doddapaneni, L. Lewis, R. Ruth, Q. Meng, V. Vee, Y. Han, J. Jayaseelan, A. English, J. Korlach, M. Hunkapiller, B. Hüttel and R. Reinhardt. We acknowledge the Yale University Biomedical High-Performance Computing Center and high-performance compute infrastructure made available through the EMBL and EMBL-EBI IT facilities. We thank the people generously contributing samples to the 1000 Genomes Project. Funding for this research project came from the following grants: NIH U41HG007497 (to C.L., E.E.E., J.O.K., M.A.B., M.G., S.A.M., R.E.M. and J.S.), RO1GM59290 (M.A.B.), R01HG002898 (S.E.D.) and R01CA166661 (S.E.D.), P01HG007497 (to E.E.E.), R01HG007068 (to R.E.M.), RR19895 and RR029676-01 (to M.B.G.), Wellcome Trust WT085532/Z/08/Z and WT104947/Z/14/Z (to P.F.), an Emmy Noether Grant from the German Research Foundation (KO4037/1-1, to J.O.K.) and the European Molecular Biology Laboratory. C.L. is on the scientific advisory board (SAB) of BioNano Genomics. E.E.E. is.on the scientific advisory board (SAB) of DNAnexus, Inc. and is a consultant for Kunming University of Science and Technology (KUST) as part of the 1000 China Talent Program. P.F. is on the SAB of Omicia, Inc. C.L. is an Ewha Womans University Distinguished Professor. E.E.E. is an investigator of the Howard Hughes Medical Institute. J.O.K. is a European Research Council investigator.

Author Contributions SV discovery \& genotyping: R.E.H., P.H.S., T.R., E.J.G., A.Ab., K.Y., F.H., K.C., G.D., K.W., M.H.-Y.F., S.K., C.A., S.A.M., R.E.M., K.Y., M.B.G., S.E.D., E.E.E., J.O.K.; SV merging \& haplotype-integration: T.R., R.E.H.,M.H.-Y.F.,E.G.,A.Me.,S.McC.; SV validation: R.E.H.,A.Ab., G.J., M.H.-Y.F., A.M.S., M.K.K., A.Ma., S.K., M.M., M.J.P.C., S.M., P.C., S.E., J.M.K B.R., J.A.W., F.Y., T.Z., M.A.B., R.E.M., A.B., C.L., E.E.E., J.O.K.; additional analyses: A.Au., 


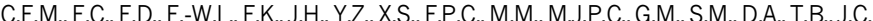
Z.C., L.D., X.F., M.G., J.M.K., H.Y.K.L., Y.K., X.J.M., B.J.N., A.N., R.A.G., M.P., M.R., R.S., D.M.M M.W., N.F.P., A.Q., E.E.S., A.S., A.A.S., A.U., C.Z., J.Z., W.Z., J.S., O.S.; data management \& archiving: L.C., X.Z.-B., P.F.; display items: P.H.S., T.R., E.J.G., A.A., Y.Z., J.H., M.H.-Y.F., K.Y., M.B.G., A.B., O.S., R.E.M., S.E.D., E.E.E., J.O.K.; organization of Supplementary Material: G.D., J.O.K., P.H.S., R.E.M.; SV Analysis group co-chairs: C.L., E.E.E., J.O.K.; manuscript writing: P.H.S., T.R., E.J.G., J.H., R.E.M., M.B.G., O.S., S.E.D., E.E.E., J.O.K.

Author Information Sequencing data, archive accessions and supporting datasets including GRCh37 variant call files comprising the extended SV Analysis Group release set, a 'readme' describing differences to the phase 3 marker paper variant release $\mathrm{e}^{16}$ and a GRCh38 version of our callset, are available at http://www.1000genomes.org/ phase-3-structural-variant-dataset. DGV archive accession: estd219. Reprints and permissions information is available at www.nature.com/reprints. The authors declare competing financial interests: details are available in the online version of the paper. Readers are welcome to comment on the online version of the paper. Correspondence and requests for materials should be addressed to E.E.E. (eee@gs.washington.edu) or J.O.K. (korbel@embl.de).

This work is licensed under a Creative Commons AttributionNonCommercial-ShareAlike 3.0 Unported licence. The images or other third party material in this article are included in the article's Creative Commons licence, Creative Commons licence, users will need to obtain permission from the licence holder to reproduce the material. To view a copy of this licence, visit http://creativecommons. org/licenses/by-nc-sa/3.0

Peter H. Sudmant ${ }^{1 *}$, Tobias Rausch ${ }^{2 *}$, Eugene J. Gardner ${ }^{3 *}$, Robert E. Handsaker ${ }^{4,5 *}$ Alexej Abyzov6*, John Huddleston ${ }^{1,7 *}$, Yan Zhang ${ }^{8,9 *}$, Kai Ye $^{10,11 *}$, Goo Jun ${ }^{12,13}$ Markus Hsi-Yang Fritz ${ }^{2}$, Miriam K. Konkel ${ }^{14}$, Ankit Malhotra ${ }^{15}$, Adrian M. Stütz ${ }^{2}$, Xinghua Shi ${ }^{16}$, Francesco Paolo Casale ${ }^{17}$, Jieming Chen ${ }^{8}, 18$, Fereydoun Hormozdiari $^{1}$, Gargi Dayama ${ }^{19}$, Ken Chen ${ }^{20}$, Maika Malig ${ }^{1}$, Mark J. P. Chaisson ${ }^{1}$, Klaudia Walter ${ }^{21}$, Sascha Meiers ${ }^{2}$, Seva Kashin ${ }^{4,5}$, Erik Garrison ${ }^{22}$, Adam Auton ${ }^{23}$, Hugo Y. K. Lam², Xinmeng Jasmine $\mathrm{Mu}^{8,25}$, Can Alkan ${ }^{26}$, Danny Antaki ${ }^{27}$, Taejeong Bae ${ }^{6}$, Eliza Cerveira ${ }^{15}$, Peter Chines ${ }^{28}$, Zechen Chong ${ }^{20}$, Laura Clarke ${ }^{17}$, Elif Dal ${ }^{26}$, Li Ding ${ }^{10,11,29,30}$, Sarah Emery ${ }^{31}$, Xian Fan ${ }^{20}$, Madhusudan Gujral ${ }^{27}$, Fatma Kahveci ${ }^{26}$, Jeffrey M. Kidd ${ }^{12,31}$, Yu Kong ${ }^{23}$, Eric-Wubbo Lameijer ${ }^{32}$, Shane McCarthy ${ }^{21}$, Paul Flicek $^{17}$, Richard A. Gibbs ${ }^{33}$, Gabor Marth ${ }^{22}$, Christopher E. Mason ${ }^{34,35}$, Androniki Menelaou $^{36,37}$, Donna M. Muzny ${ }^{38}$, Bradley J. Nelson ${ }^{1}$, Amina Noor ${ }^{27}$, Nicholas F. Parrish $^{39}$, Matthew Pendleton ${ }^{38}$, Andrew Quitadamo ${ }^{16}$, Benjamin Raeder ${ }^{2}$, Eric E. Schadt ${ }^{38}$, Mallory Romanovitch ${ }^{15}$, Andreas Schlattl ${ }^{2}$, Robert Sebra ${ }^{38}$, Andrey A. Shabalin ${ }^{40}$, Andreas Untergasser ${ }^{2,41}$, Jerilyn A. Walker ${ }^{14}$, Min Wang ${ }^{33}$, Fuli Yu ${ }^{33}$ Chengsheng Zhang ${ }^{15}$, Jing Zhang ${ }^{8,9}$, Xiangqun Zheng-Bradley ${ }^{17}$, Wanding Zhou' ${ }^{20}$, Thomas Zichner ${ }^{2}$, Jonathan Sebat ${ }^{27}$, Mark A. Batzer ${ }^{14}$, Steven A. McCarroll ${ }^{4,5}$, The 1000 Genomes Project Consortium $\dagger$, Ryan E. Mills ${ }^{19,31} \S$, Mark B. Gerstein ${ }^{8,9,42} \S$, Ali Bashir ${ }^{38} \S$, Oliver Stegle ${ }^{17} \S$, Scott E. Devine ${ }^{3} \S$, Charles Lee ${ }^{15,43} \S$, Evan E. Eichler ${ }^{1,7} \S$ \& Jan O. Korbe ${ }^{2,17} \S$

${ }^{1}$ Department of Genome Sciences, University of Washington, 3720 15th Avenue NE, Seattle, Washington 98195-5065, USA. ${ }^{2}$ European Molecular Biology Laboratory (EMBL), Genome Biology Unit, Meyerhofstrasse 1, 69117 Heidelberg, Germany. ${ }^{3}$ Institute for Genome Sciences, University of Maryland School of Medicine, 801 W Baltimore Street, Baltimore, Maryland 21201, USA. ${ }^{4}$ Department of Genetics, Harvard Medical School, Boston, 25 Shattuck Street, Boston, Massachusetts 02115, USA. ${ }^{5}$ Program in Medical and Population Genetics, Broad Institute of MIT and Harvard, 415 Main Street, Cambridge, Massachusetts 02142, USA. ${ }^{6}$ Department of Health Sciences Research, Center for Individualized Medicine, Mayo Clinic, 200 First Street SW, Rochester, Minnesota 55905
USA. ${ }^{7}$ Howard Hughes Medical Institute, University of Washington, Seattle, Washington 98195, USA. ${ }^{8}$ Program in Computational Biology and Bioinformatics, Yale University, BASS 432 \& 437, 266 Whitney Avenue, New Haven, Connecticut 06520, USA.

${ }^{9}$ Department of Molecular Biophysics and Biochemistry, School of Medicine, Yale University, 266 Whitney Avenue, New Haven, Connecticut 06520, USA. ${ }^{10}$ The Genome Institute, Washington University School of Medicine, 4444 Forest Park Avenue, St Louis, Missouri 63108, USA. ${ }^{11}$ Department of Genetics, Washington University in St Louis, 4444 Forest Park Avenue, St Louis, Missouri 63108, USA. ${ }^{12}$ Department of Biostatistics and Center for Statistical Genetics, University of Michigan, 1415 Washington Heights, Ann Arbor, Michigan 48109, USA. ${ }^{13}$ Human Genetics Center, School of Public Health, The University of Texas Health Science Center at Houston, 1200 Pressler St., Houston, Texas 77030, USA. ${ }^{14}$ Department of Biological Sciences, Louisiana State University, 202 Life Sciences Building, Baton Rouge, Louisiana 70803, USA. ${ }^{15}$ The Jackson Laboratory for Genomic Medicine, 10 Discovery 263 Farmington Avenue, Farmington, Connecticut 06030, USA. ${ }^{16}$ Department of Bioinformatics and Genomics, University of North Carolina at Charlotte, 9201 University City Blvd., Charlotte, North Carolina 28223, USA.

${ }^{17}$ European Molecular Biology Laboratory, European Bioinformatics Institute (EMBL-EBI), Wellcome Trust Genome Campus, Hinxton, Cambridge CB10 1SD, UK. ${ }^{18}$ Integrated Graduate Program in Physical and Engineering Biology, Yale University, New Haven, Connecticut 06520, USA. ${ }^{19}$ Department of Computational Medicine \& Bioinformatics, University of Michigan, $500 \mathrm{~S}$. State Street, Ann Arbor, Michigan 48109, USA. ${ }^{20}$ The University of Texas MD Anderson Cancer Center, 1515 Holcombe Boulevard, Houston, Texas 77030, USA. ${ }^{21}$ The Wellcome Trust Sanger Institute, Wellcome Trust Genome Campus, Hinxton, Cambridge CB10 1SA, UK. ${ }^{22}$ Department of Biology, Boston College, 355 Higgins Hall, 140 Commonwealth Avenue, Chestnut Hill, Massachusetts 02467, USA ${ }^{23}$ Department of Genetics, Albert Einstein College of Medicine, 1301 Morris Park Avenue, Bronx, New York 10461, USA. ${ }^{24}$ Bina Technologies, Roche Sequencing, 555 Twin Dolphin Drive, Redwood City, California 94065, USA. ${ }^{25} \mathrm{Cancer}$ Program, Broad Institute of MIT and Harvard, 415 Main Street, Cambridge, Massachusetts 02142, USA. ${ }^{26}$ Department of Computer Engineering, Bilkent University, 06800 Ankara, Turkey. ${ }^{27}$ University of California San Diego (UCSD), 9500 Gilman Drive, La Jolla, California 92093, USA.

${ }^{28}$ National Human Genome Research Institute, National Institutes of Health, Bethesda, Maryland 20892 USA. ${ }^{29}$ Department of Medicine, Washington University in St Louis, 4444 Forest Park Avenue, St Louis, Missouri 63108, USA. ${ }^{30}$ Siteman Cancer Center, 660 South Euclid Avenue, St Louis, Missouri 63110, USA. ${ }^{31}$ Department of Human Genetics, University of Michigan, 1241 Catherine Street, Ann Arbor, Michigan 48109, USA. ${ }^{32}$ Molecular Epidemiology, Leiden University Medical Center, Leiden 2300RA, The Netherlands. ${ }^{33}$ Baylor College of Medicine, 1 Baylor Plaza, Houston, Texas 77030, USA. ${ }^{34}$ The Department of Physiology and Biophysics and the HRH Prince Alwaleed Bin Tala Bin Abdulaziz Alsaud Institute for Computational Biomedicine, 1305 York Avenue, Weil Cornell Medical College, New York, New York 10065, USA. ${ }^{35}$ The Feil Family Brain and Mind Research Institute, 413 East 69th St, Weill Cornell Medical College, New York, New York 10065, USA. ${ }^{36}$ University of Oxford, 1 South Parks Road, Oxford OX3 9DS, UK.

${ }^{37}$ Department of Medical Genetics, Center for Molecular Medicine, University Medical Center Utrecht, Utrecht, 3584 CG, The Netherlands. ${ }^{38}$ Department of Genetics and Genomic Sciences, Icahn School of Medicine, New York School of Natural Sciences, 1428 Madison Avenue, New York, New York 10029, USA. ${ }^{39}$ Institute for Virus Research, Kyoto University, 53 Shogoin Kawahara-cho, Sakyo-ku, Kyoto 606-8507, Japan. ${ }^{40}$ Center for Biomarker Research and Precision Medicine, Virginia Commonwealth University, 1112 East Clay Street, McGuire Hall, Richmond, Virginia 23298-0581, USA. ${ }^{41}$ Zentrum für Molekulare Biologie, University of Heidelberg, Im Neuenheimer Feld 282, 69120 Heidelberg, Germany. ${ }^{42}$ Department of Computer Science, Yale University, 51 Prospect Street, New Haven, Connecticut 06511. USA. ${ }^{43}$ Department of Graduate Studies - Life Sciences, Ewha Womans University, Ewhayeodae-gil, Seodaemun-gu, Seoul 120-750, South Korea.

*These authors contributed equally to this work.

$\dagger$ A list of participants and their affiliations appears in the Supplementary Information. $\S$ These authors jointly supervised this work. 
a

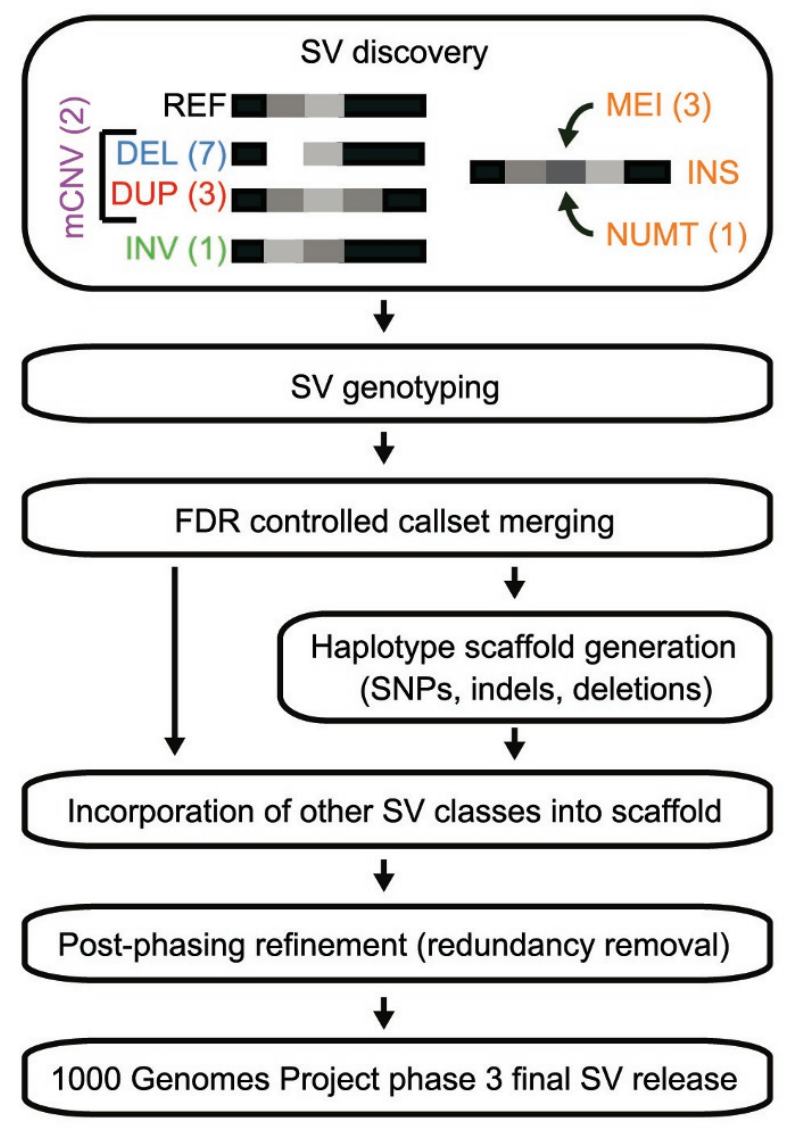

b
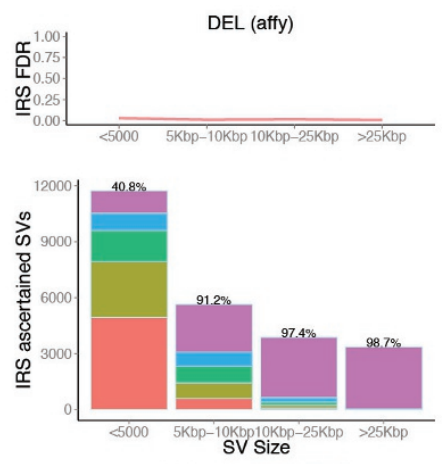

\#Probes $\square 1 \square 2 \llbracket 3 \llbracket 4 \llbracket>4$

C
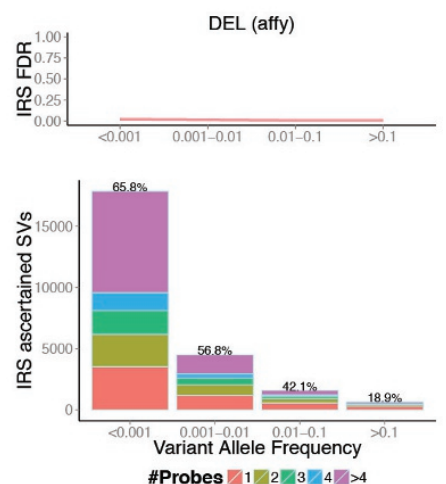

d
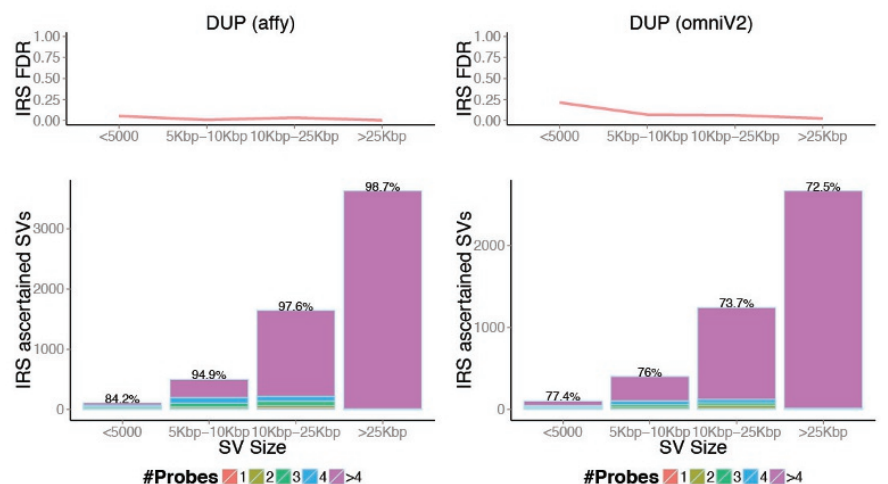

e
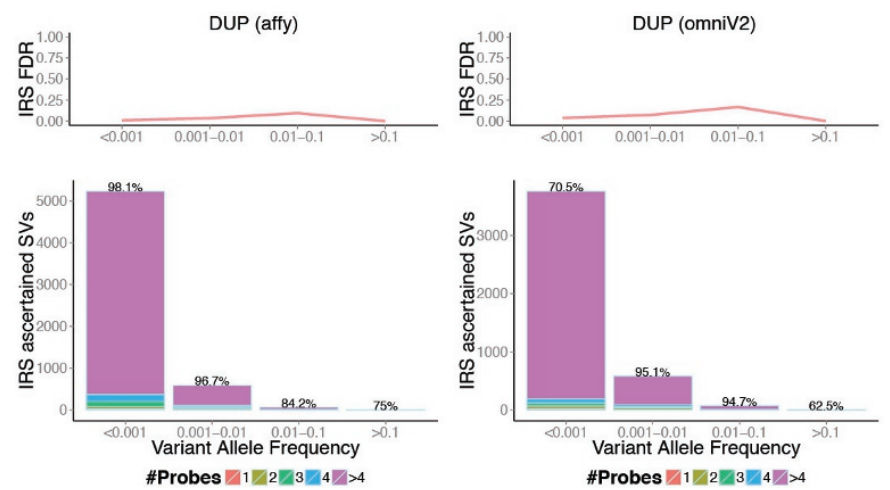

d, IRS results for duplications in different size bins. e, IRS validation results for duplications in VAF bins. Based on Affymetrix SNP6 array probes, the IRS FDR for all SV length and VAF bins was $\leq 5.4 \%$, requiring at least $100 \mathrm{SVs}$ per bin with an IRS assigned $P$-value.
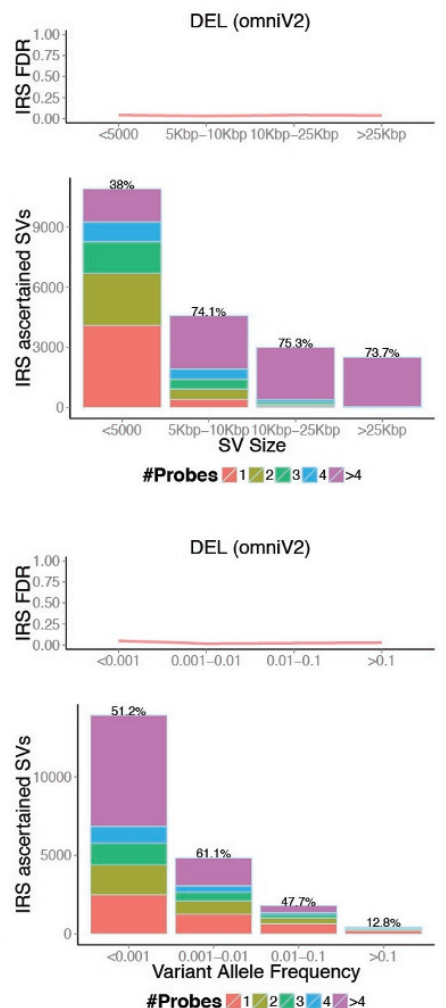

\#Probes $\forall 1 \square 2 \square 3 \square 4 \square>0$
Extended Data Figure $1 \mid$ Construction of the SV release and intensity
sum validation. a, Approach used for constructing our SV release set.

b, Intensity rank sum (IRS) validation results for deletions in different size bins. c, IRS validation results for deletions in variant allele frequency (VAF) bins.

. 

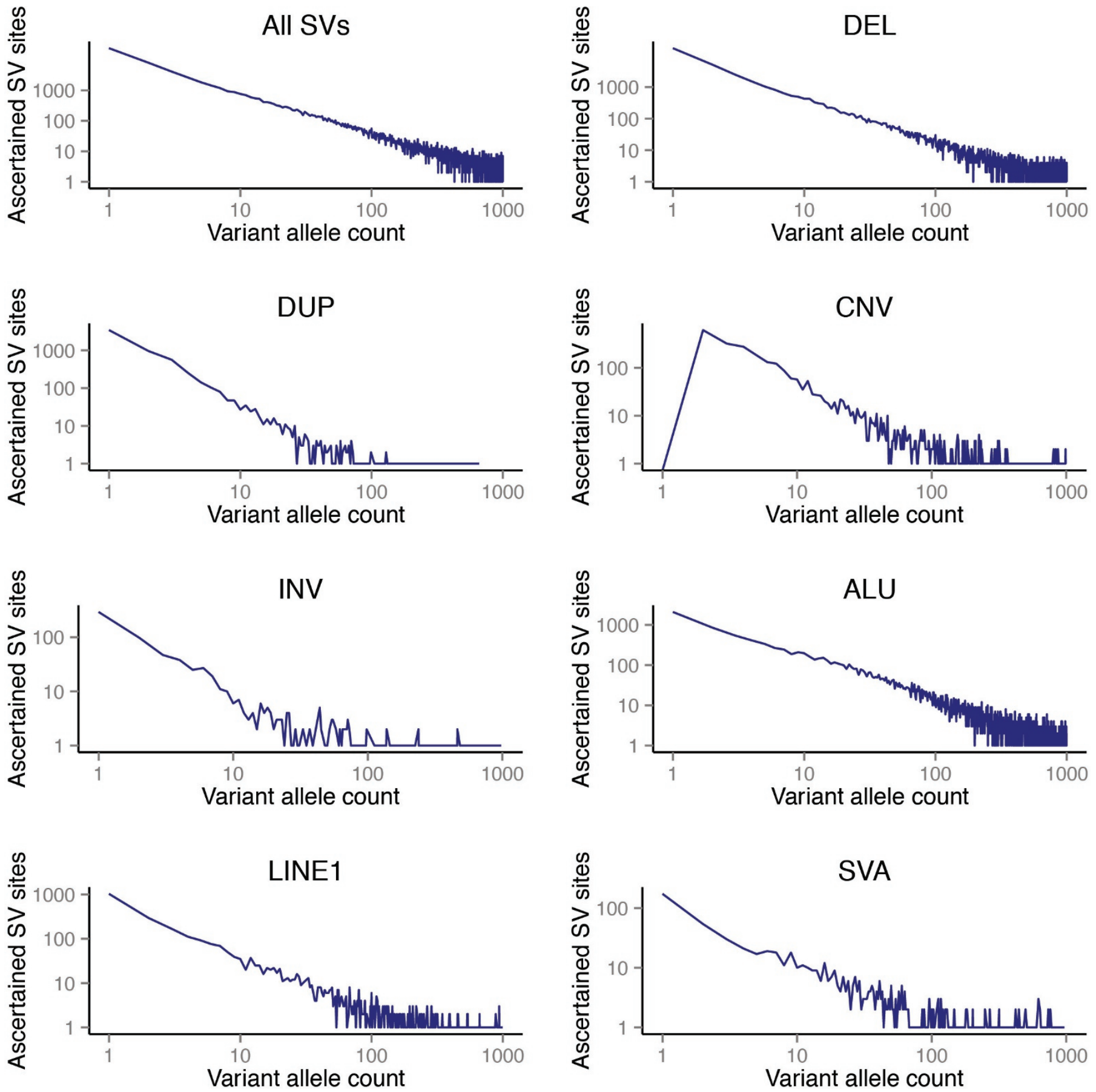

Extended Data Figure $2 \mid$ This figure shows the number of SV sites in our phase 3 release relative to allele frequency expressed in terms of allele count. SVs down to an allele count of 1 (corresponding to VAF $=0.0002$ ) are represented in our phase $3 \mathrm{SV}$ set (with the exception of $\mathrm{mCNVs}$, denoted

'CNV' in this figure, which are defined as sites of multi-allelic variation thus requiring allele count $\geq 2$, hence no mCNV sites are ascertained for allele count $=1$ ) 
a
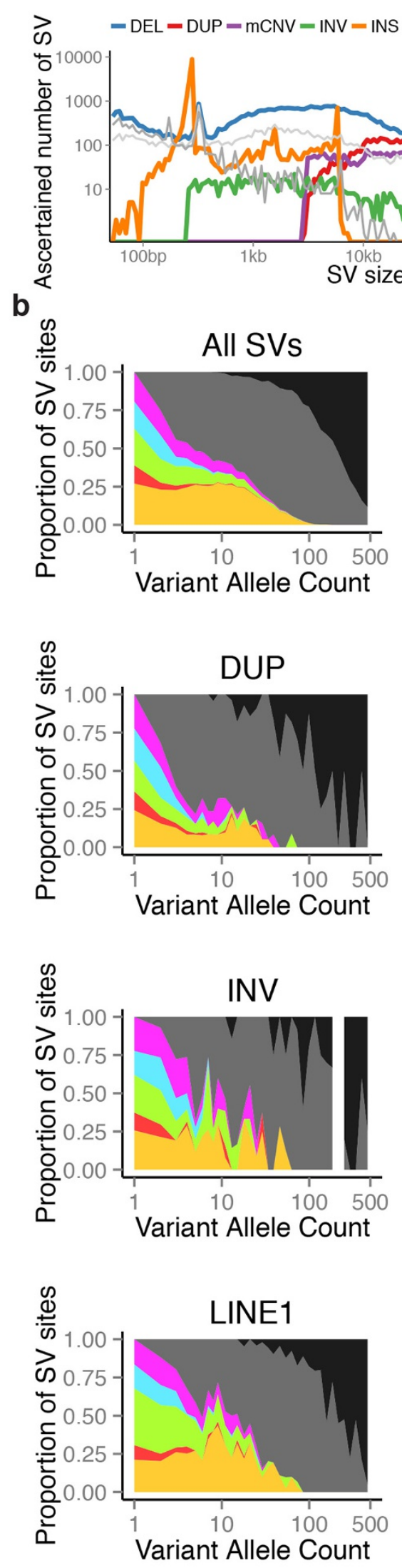

DEL_PILOT - DEL_CHM1

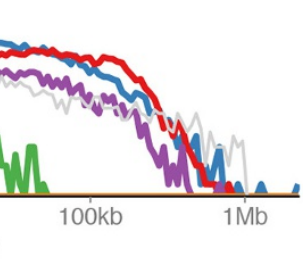

Superpopulation

\section{Superpopulation}

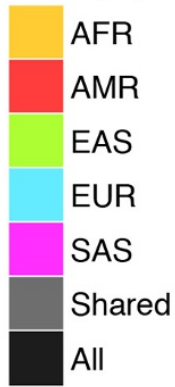

Superpopulation

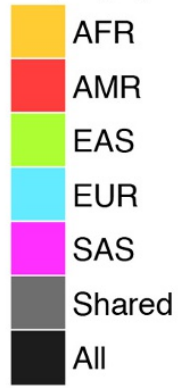

Superpopulation

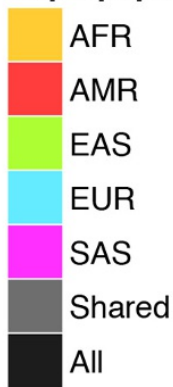

C
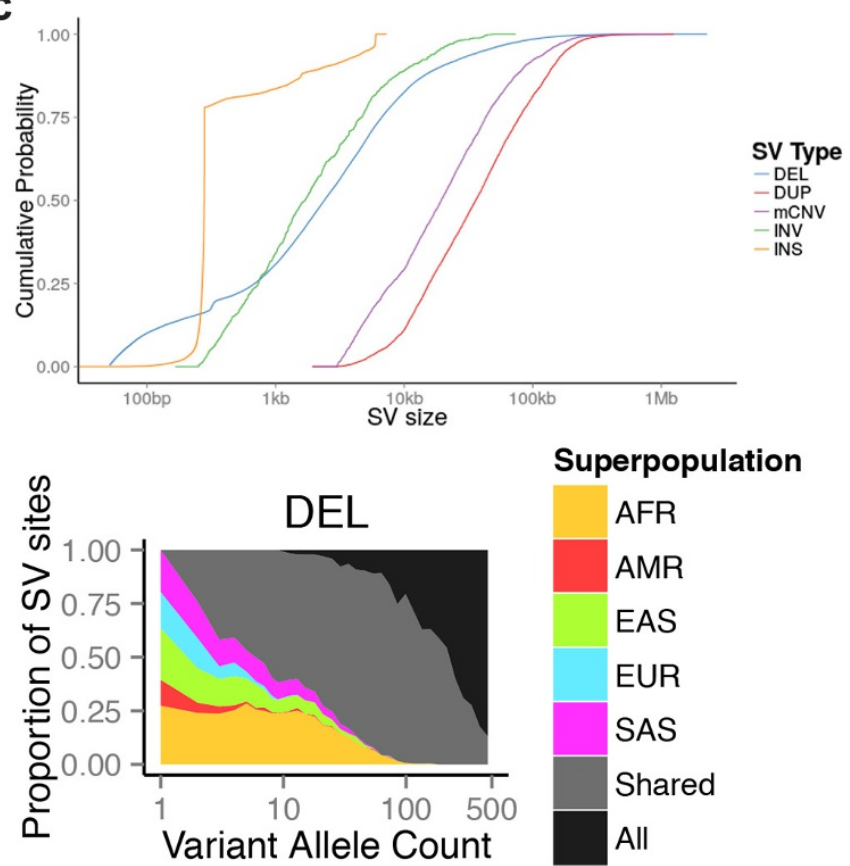

Superpopulation

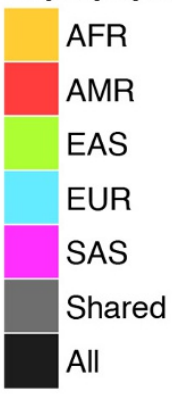

\section{Superpopulation}
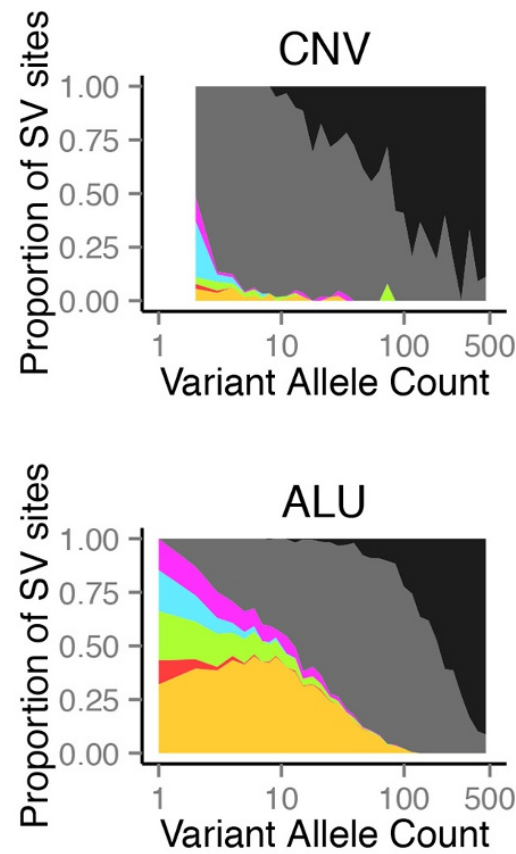

Superpopulation

Superpopulation

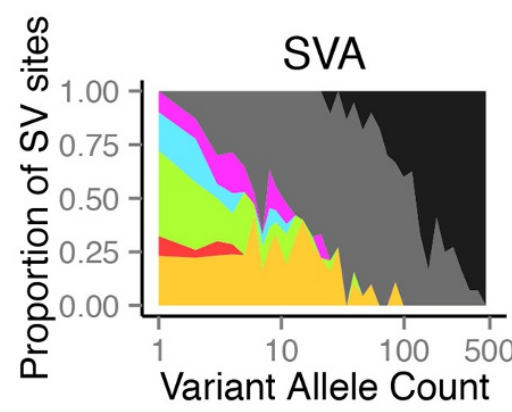

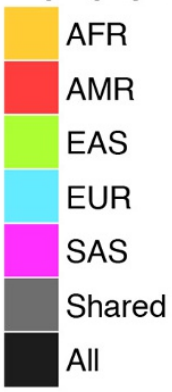
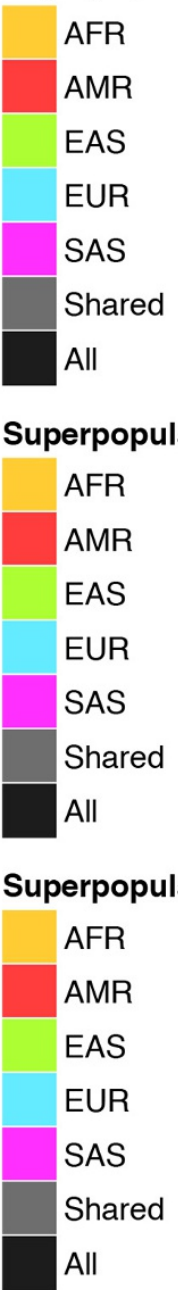
Extended Data Figure $3 \mid$ Size and population distribution of different SV classes. a, Variants ascertained in the 1000 Genomes Project pilot phase ${ }^{6}$ (light grey) as well as the recent publication of SVs ascertained by PacBio sequencing in the CHM1 genome ${ }^{14}$ (grey) are displayed for comparison in this SV size distribution figure (INS, used as abbreviation for MEIs and NUMTs in this display item). b. Population distribution of SV allele sharing across continental groups for different SV classes. c, Cumulative distributions of the number of events as a function of size by SV class. 
a
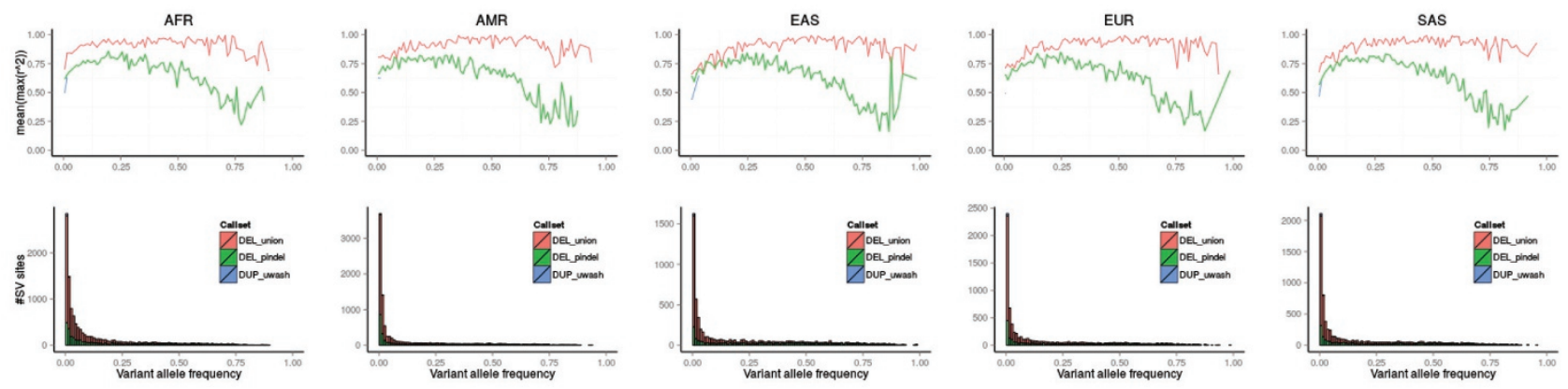

b
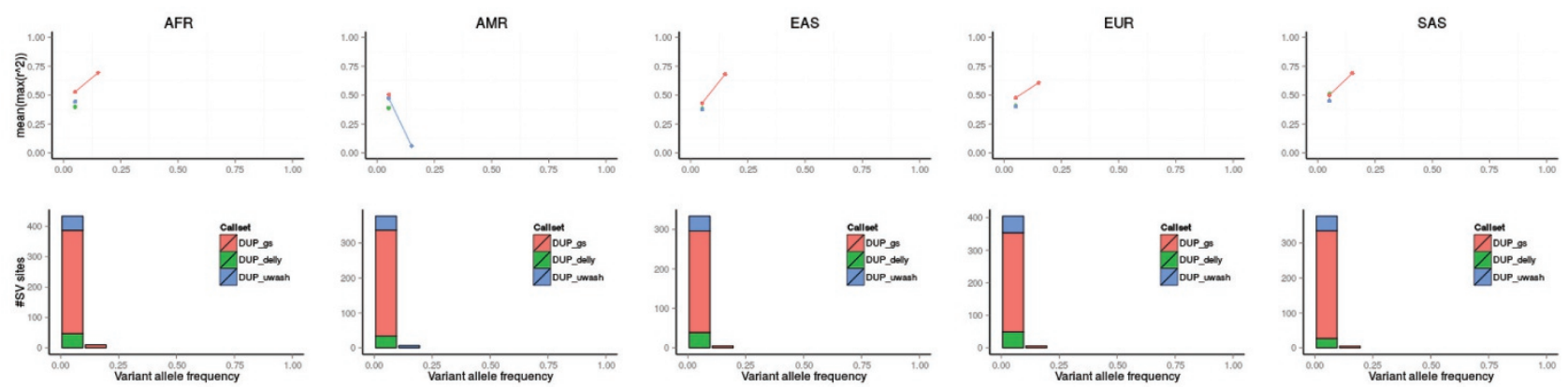

C
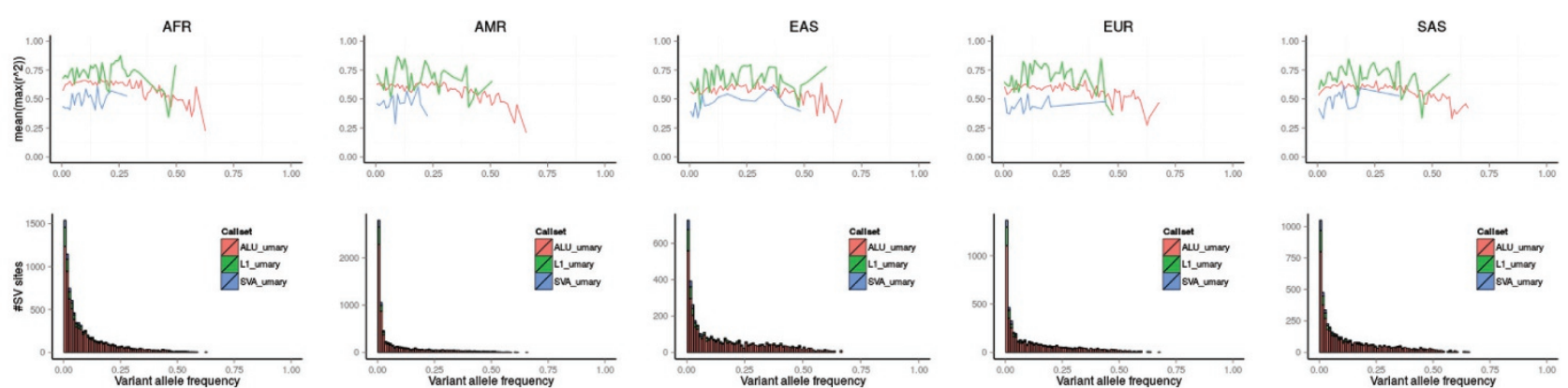

d
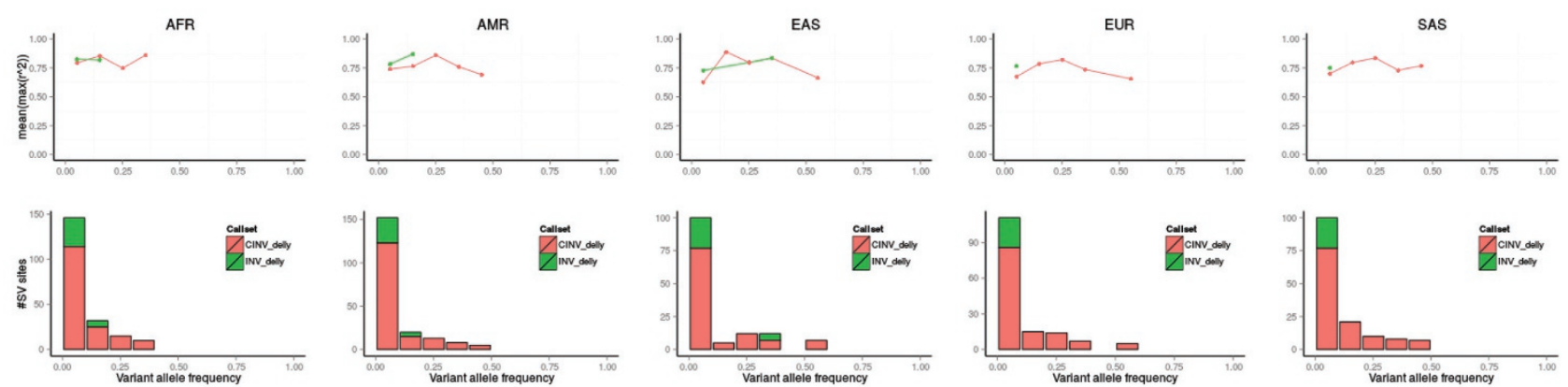

Extended Data Figure $4 \mid$ LD properties of various SV classes. a, LD properties of deletions, broken down by continental group and shown as a function of VAF. b, LD properties of duplications. c, LD properties of $A l u, \mathrm{~L} 1$ and SVA mobile element insertions. d, LD properties of inversions (with discovery algorithm Delly; that is, CINV = one-sided inversions with support for one breakpoint; INV = two-sided inversions with support for both breakpoints; these two sets are combined into the joint phase 3 SV group breakdown for two independent inversion sets generated with our inversion inversion set). 
a

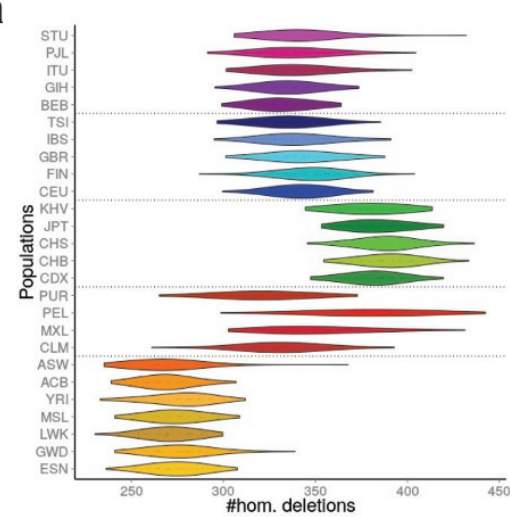

C

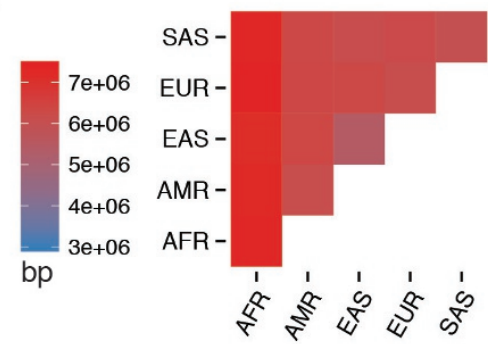

deletions
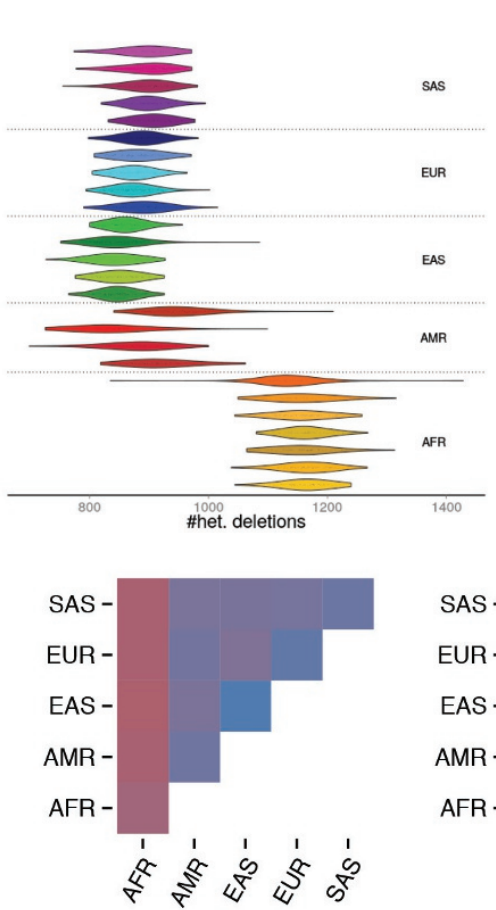

SNPS b

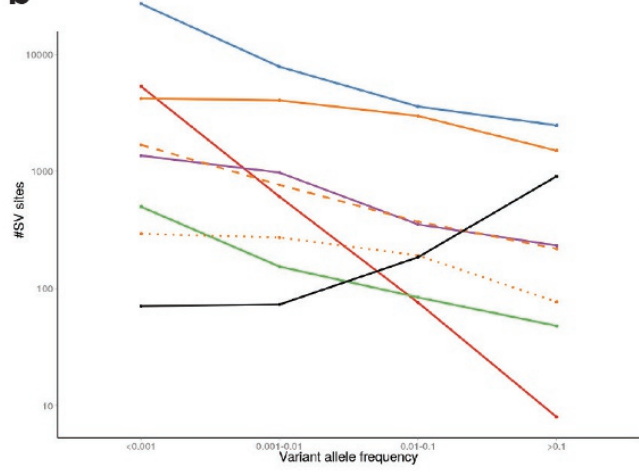

d

$59 ; 5 ; 4$

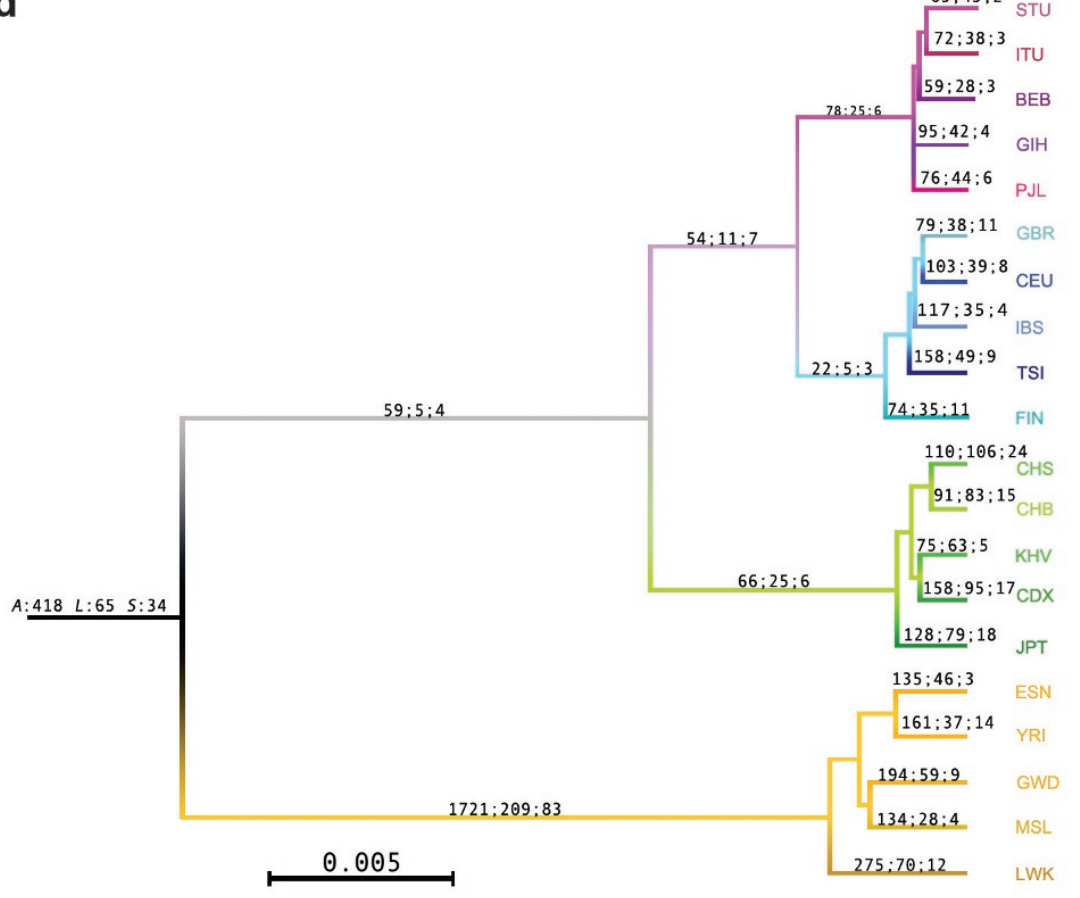

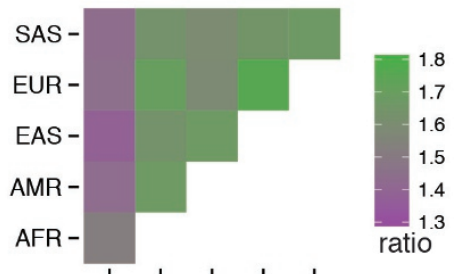

क्षे

deletion bp / SNP bp

\section{e}

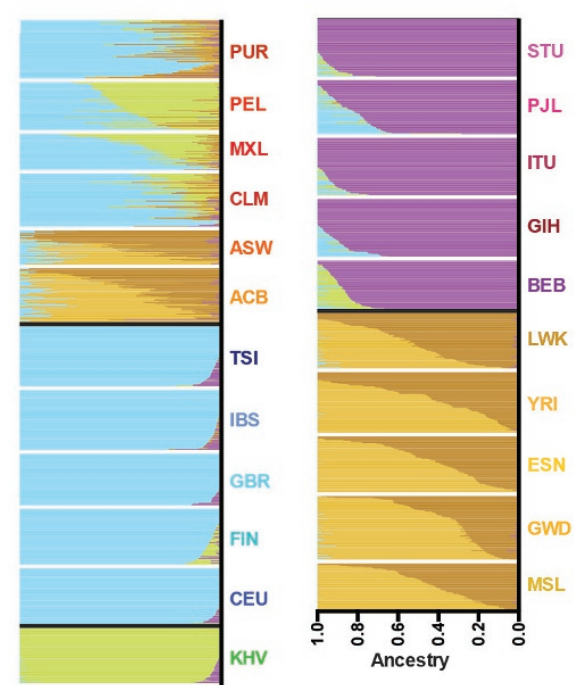




\section{RESEARCH ARTICLE}

Extended Data Figure $5 \mid$ Population genetic properties of SVs. a, Deletion heterozygosity and homozygosity among human populations for a subset of high-confidence deletions. Populations from the African continental group (AFR) exhibit the highest levels of heterozygosity and thus diversity among humans, but show the overall lowest level of deletion homozygosity among all continental groups. By comparison, East Asian populations exhibited the lowest levels of deletion heterozygosity and the highest levels of homozygosity. Het., heterozygous; Hom., homozygous. b, VAF distribution of major SV classes. Bi-allelic duplications represent a notable outlier, showing a striking depletion of common alleles, which can be explained by the preponderance of genomic sites of duplication to undergo recurrent rearrangement (see main text). As a consequence, most common duplications are classified as multiallelic variants (that is, $\mathrm{mCNVs}$ ). c, The number of base pairs (bp) differing among individuals within and between continental groups for deletions (upper panel) and SNPs (middle panel) contrasted with the ratio of deletion bp differences to SNP bp differences ('deletion bp/SNP bp') among groups (lower panel). Non-African groups exhibit a higher 'deletion bp/SNP bp' compared to Africans. d, Neighbour-joining tree of populations constructed from MEIs (homoplasy-free markers) to provide a (simplified) view of population ancestry. The tree is labelled with the number of lineage-specific MEIs (Alu:L1:SVA). e, Classification of ancestry in AFR/AMR and AMR admixed populations using homoplasy-free ancestry informative MEI markers. Colour usage follows the same scheme as in Fig. 1d, except in the case of AFR individuals, which use both the colour in Fig. 1d and another colour that is unrelated to any other figure to indicate additional substructure within this group. 
a

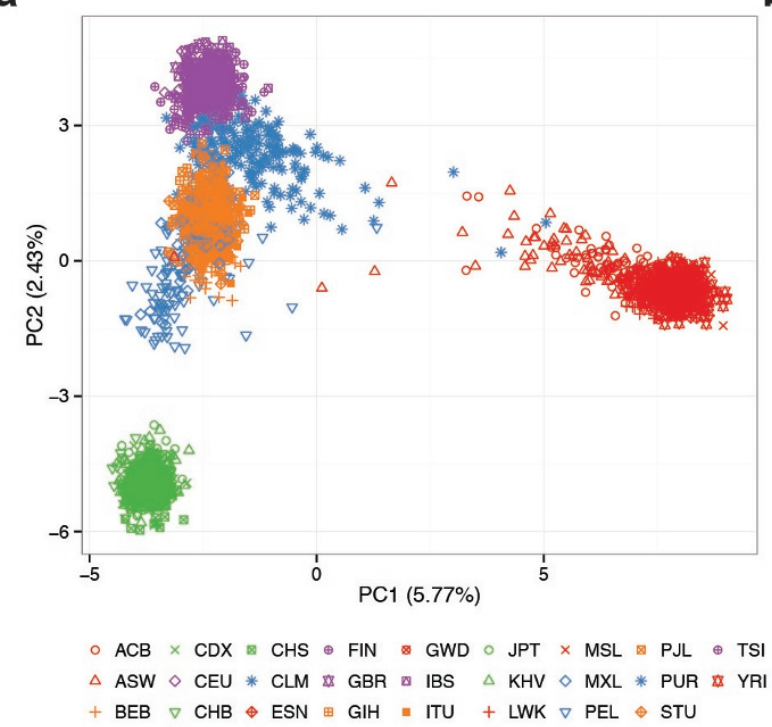

b

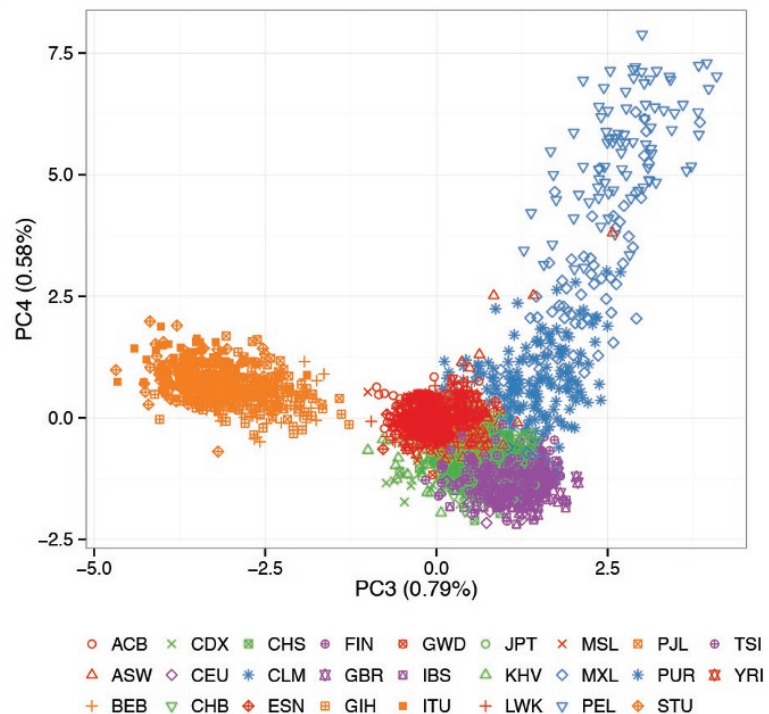

C

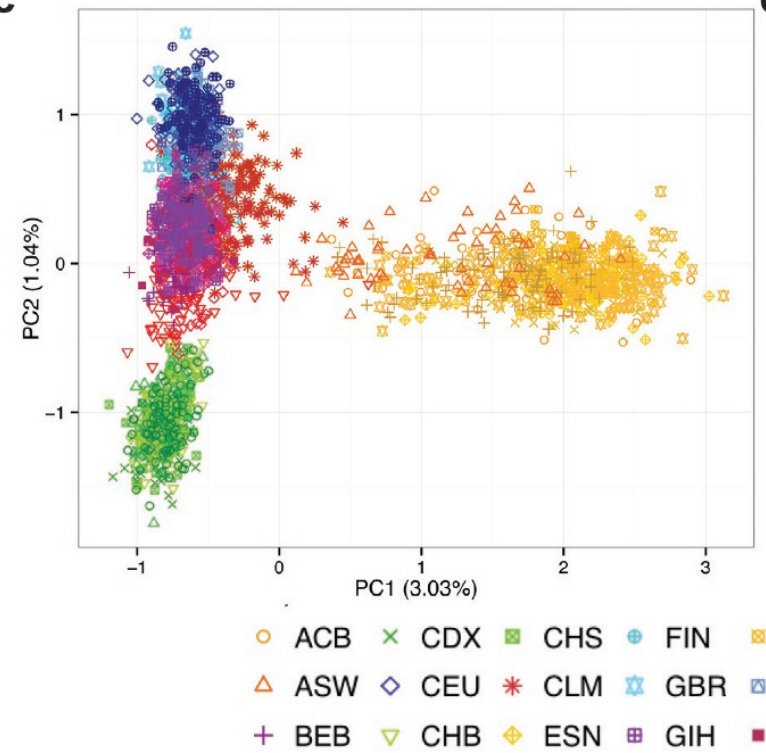

d

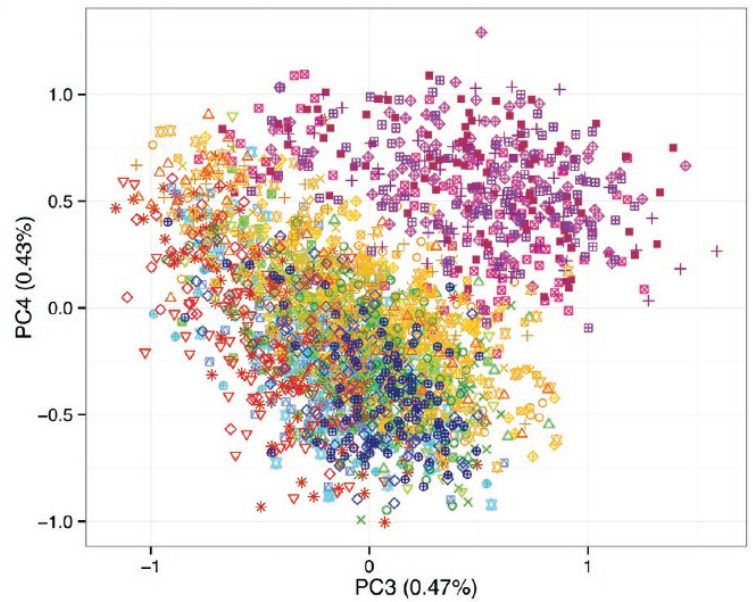

GWD $\circ \mathrm{JPT} \times \mathrm{MSL} \otimes \mathrm{PJL} \oplus \mathrm{TSI}$

IBS $\triangle \mathrm{KHV} \diamond \mathrm{MXL} *$ PUR $\& \mathrm{YRI}$

$+\mathrm{LWK} \nabla \mathrm{PEL} \oplus \mathrm{STU}$
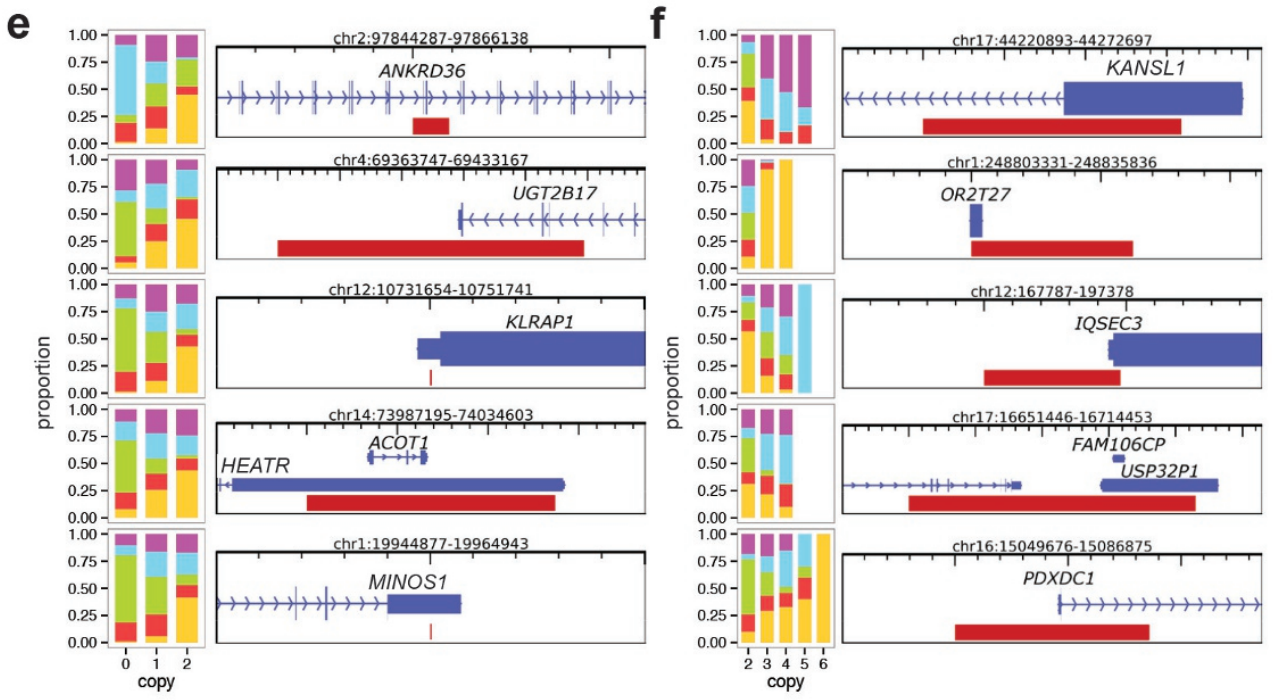

AFR $\square$ AMR $=$ EAS EUR $\square$ SAS 


\section{RESEARCH ARTICLE}

Extended Data Figure 6 Principal component analysis and population stratification of SVs. a, Principal component analysis (PCA) plot of principal components 1 and 2 for deletions. b, PCA plot of principal components 3 and 4 for deletions. c, PCA plot of principal components and 2 for MEIs. d, PCA plot of principal components 3 and 4 for MEIs. e, The five most highly population-stratified deletions intersecting proteincoding genes based on $V_{\mathrm{ST}}$. $\mathbf{f}$, The five most highly population-stratified duplications and multi-allelic copy number variants (mCNVs) intersecting protein-coding genes based on $V_{\mathrm{ST}}$. For abbreviations, see Supplementary Table 1. 

DEL overlap with genomic elements (partial overlap)

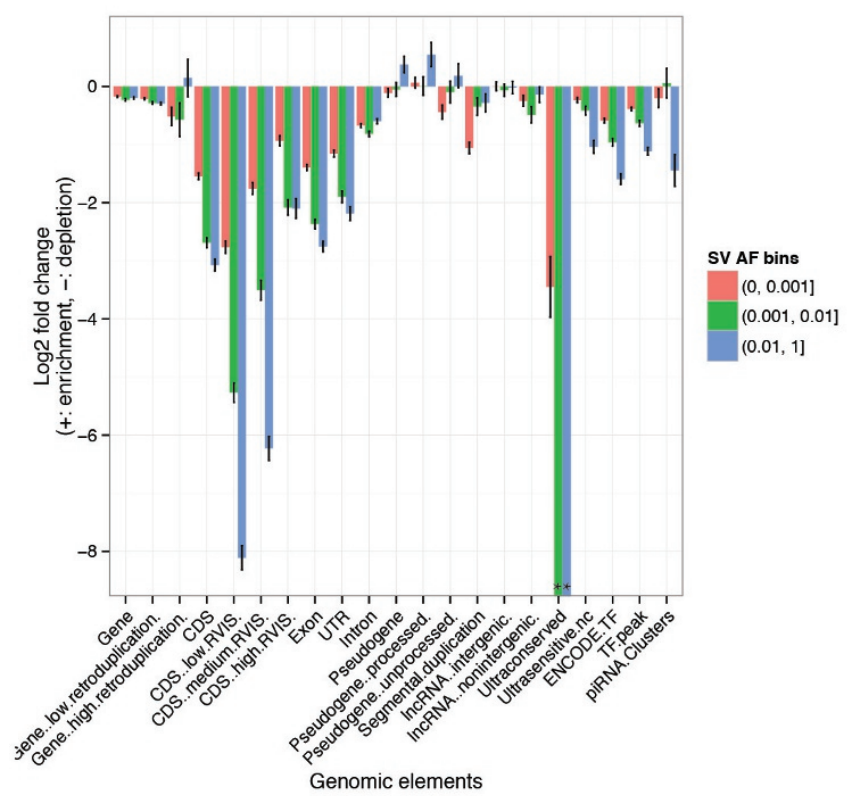

C

SNVs overlap with genomic elements (partial overlap)

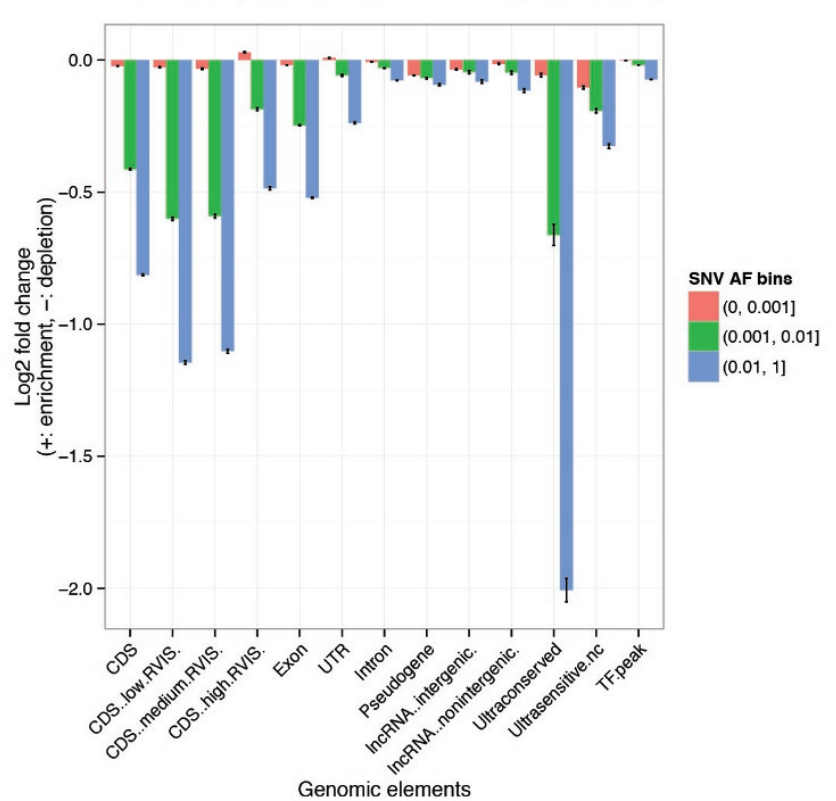

Extended Data Figure $7 \mid$ Enrichment of functional elements intersecting SVs. a, Shadow figure of Fig. 2a. Overlap enrichment analysis of deletions (with resolved breakpoints) versus genomic elements, using partial overlap statistic, deletions categorized into VAF bins. b. Similar to $\mathbf{a}$. The only difference is that engulf overlap statistic is used instead of partial overlap statistic. Engulf overlap statistic is the count of genomic elements (for example, CDS) that are fully imbedded in at least one SV interval (for example, b

DEL overlap with genomic elements (engulf overlap)

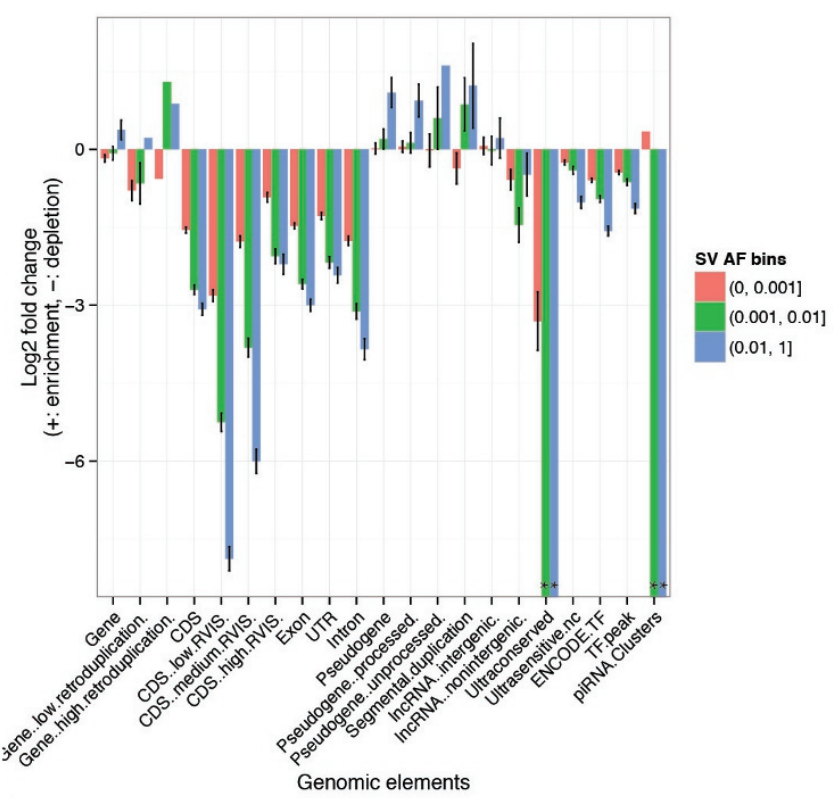

d SVs overlap with genomic elements (partial overlap)

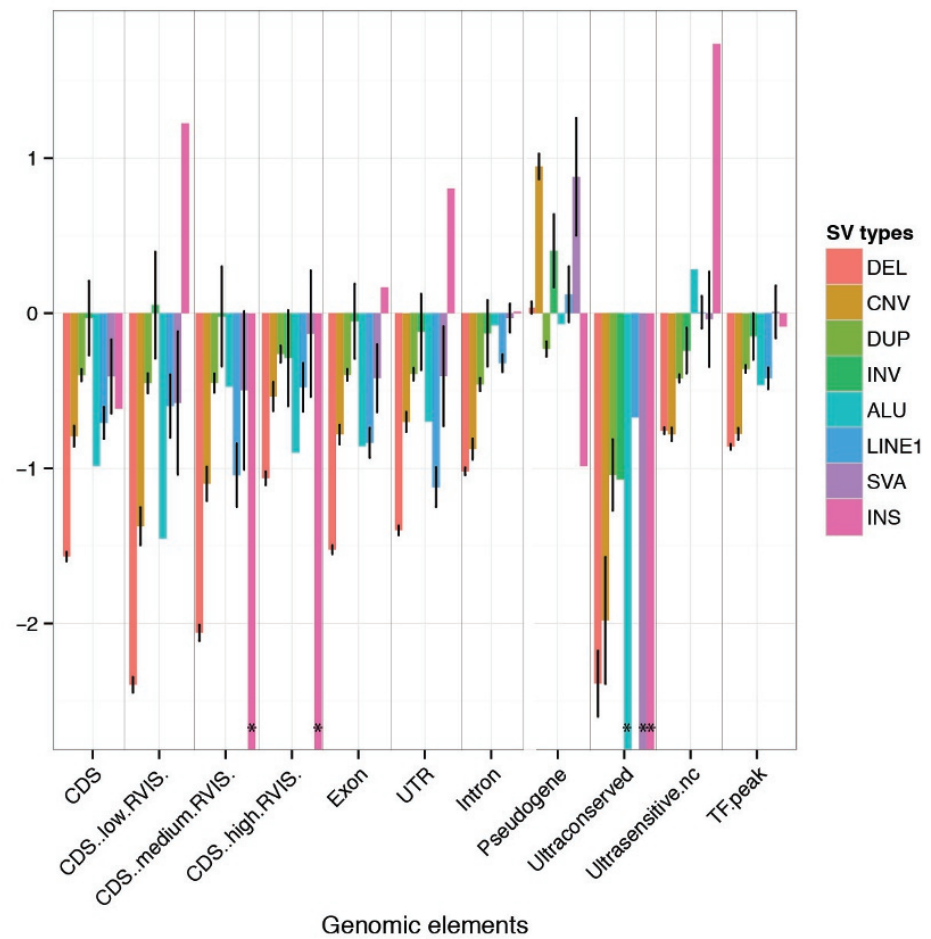

deletions). *no element intersected observed within data set. c, Similar to a and b, with the enrichment/depletion analysis pursued for common SNPs as well as more rare single nucleotide polymorphisms/variants (SNVs). Common SNV alleles show the highest levels of depletion for investigated genomic elements. d, Overlap enrichment analysis of various SV types versus genomic elements, using partial overlap statistic. 
a

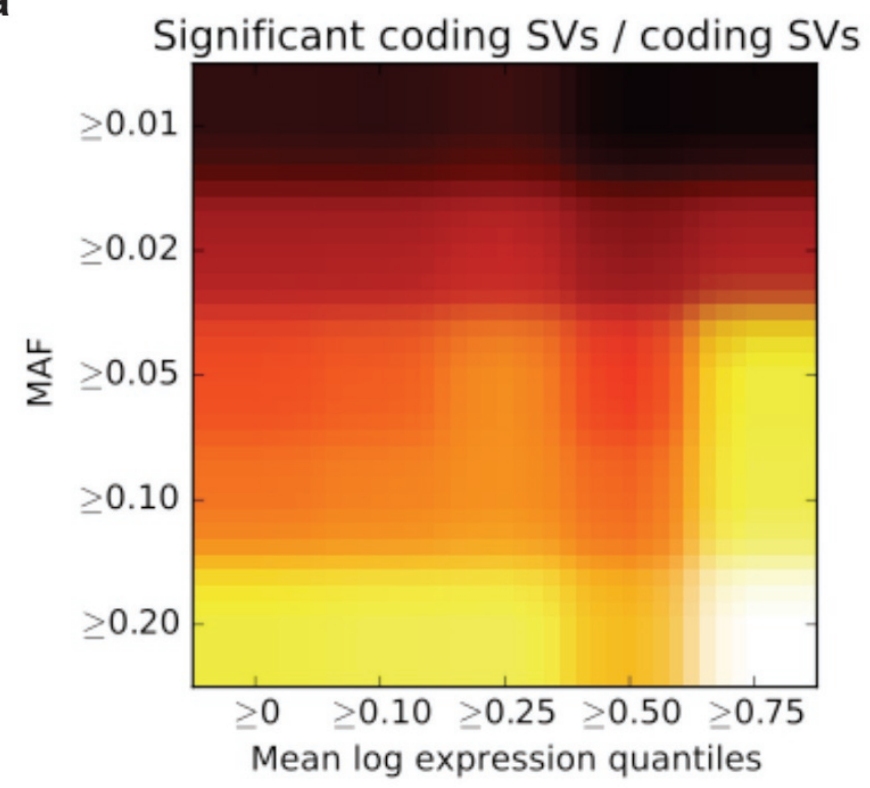

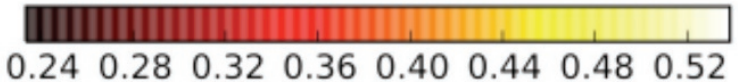

C

not significant $\quad$ Indel eQTL lead SV eQTL

- SNP eQTL $\quad$ LD tagged SV eQTL

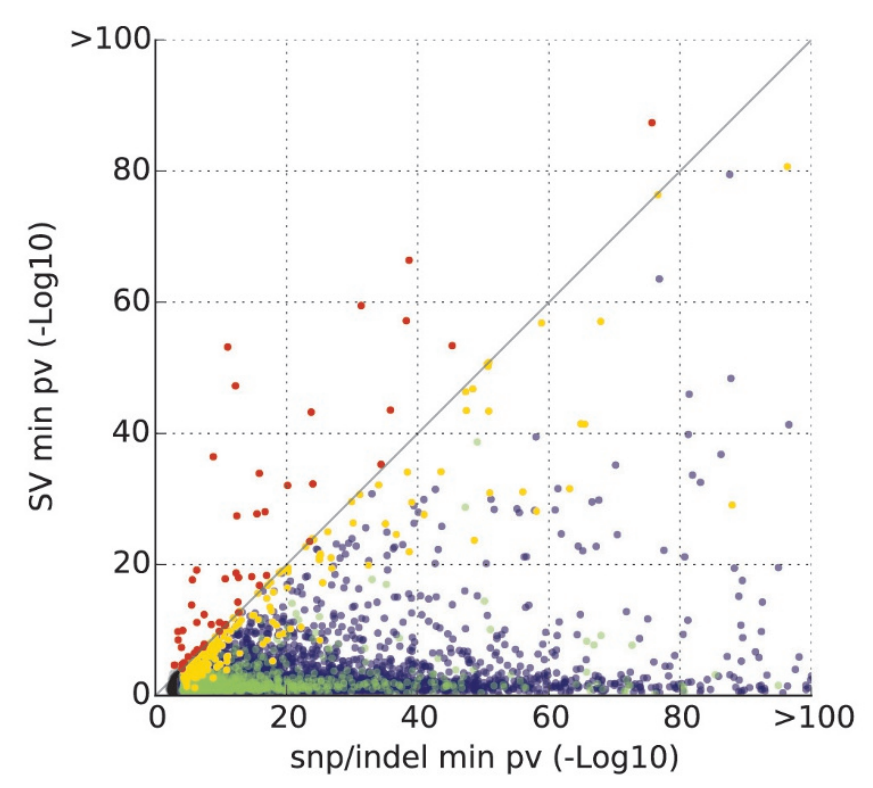

Extended Data Figure $8 \mid$ SV-eQTL analysis. a, SV-centric eQTL analysis of coding SVs. Shown is the proportion of coding SVs that are eQTLs as a function of the minimum VAF and the expression quartile. $\mathbf{b}$, Total number of coding SVs for corresponding filters. Common SVs (VAF > 0.2) in highly expressed genes ( $>75 \%$ quantile) are very likely to correspond to SV-eQTLs (54\%, see also Supplementary Table 8). c, For all genes with significant eQTLs (FDR $<10 \%$ ), shown are raw $P$-values considering only SNPs ( $x$ axis) or only SVs ( $y$ axis). Genes with (strict lead) SV-eQTLs are shown in red. Genes
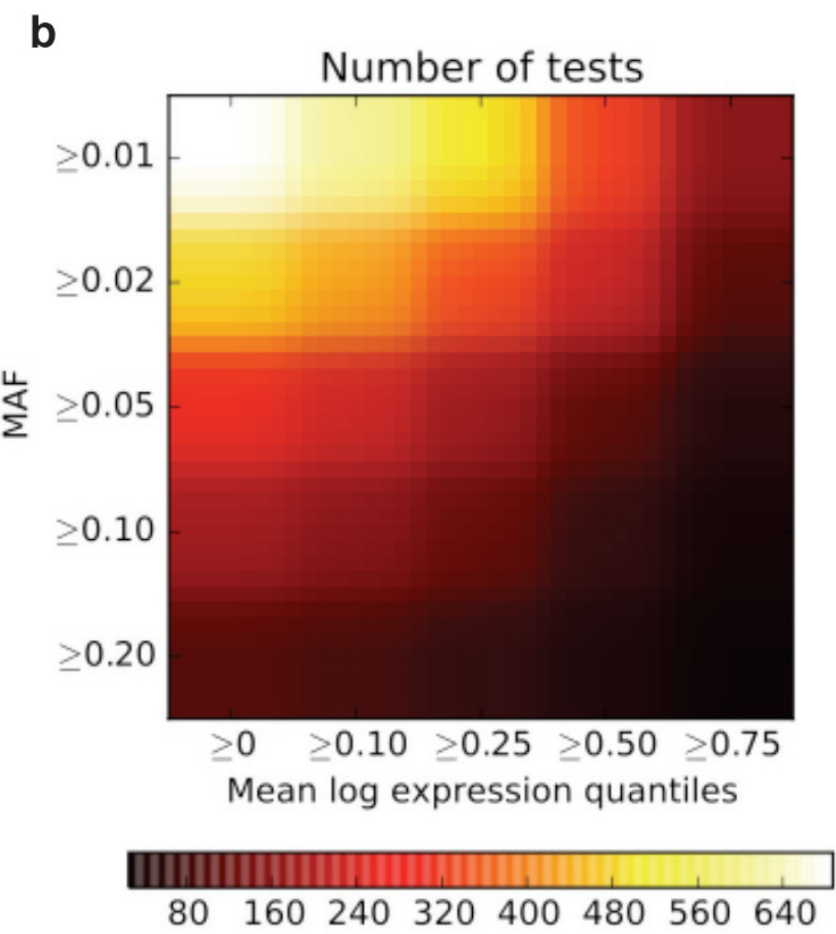

d

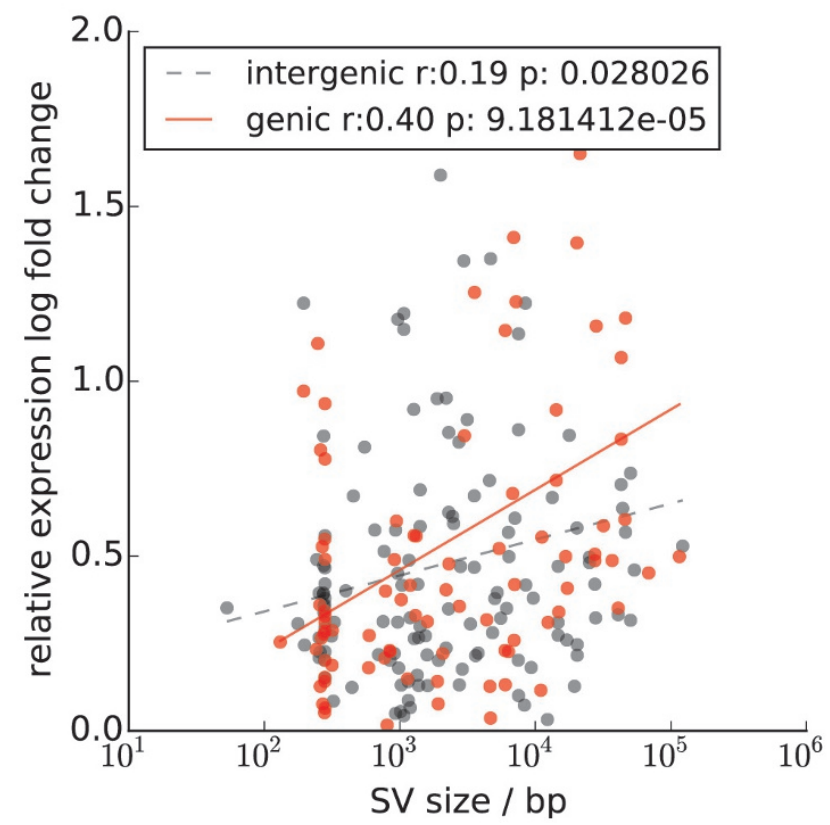

with a SNP lead eQTL that is in linkage with an SV $\left(r^{2}>0.5\right)$ are shown in orange. SNP lead eQTLs without an SV in LD are shown in blue. d, Relative eQTL effect sizes for genetic and intergenic SV eQTLs $(n=239)$ either with an SV-eQTL or an LD tagged SV (in log abundance scale). Shown are regression trends for both genic and intergenic SV eQTls. For genetic eQTLs, a clear relationship between SV effect size is found. For example, genic SVs $>10 \mathrm{~kb}$ have threefold larger effect sizes compared to genic SVs $<1 \mathrm{~kb} ; P=0.004$; $t$-test. 

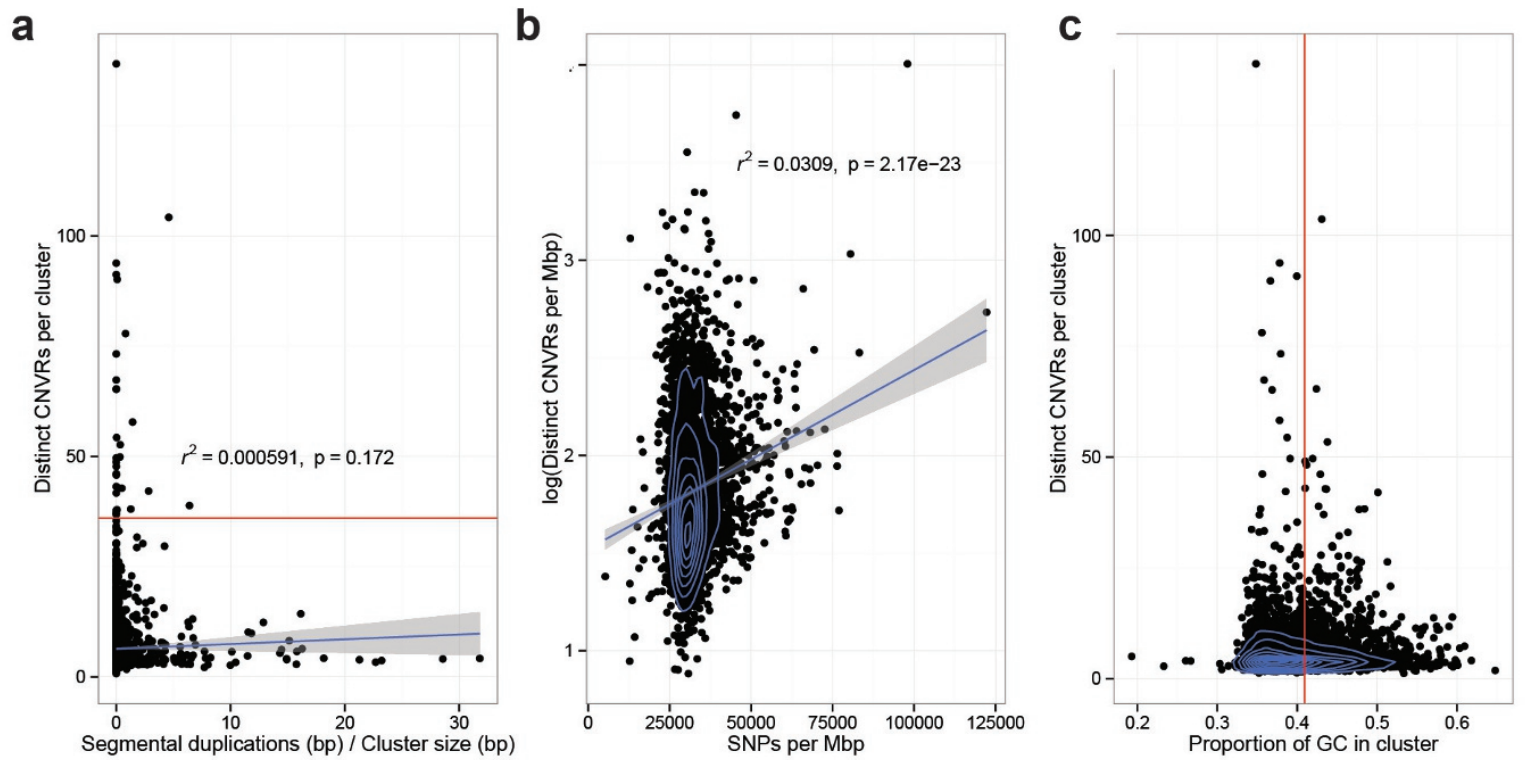

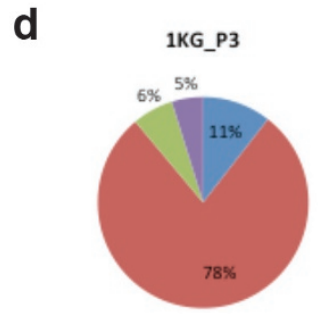

e

f Mills_2011

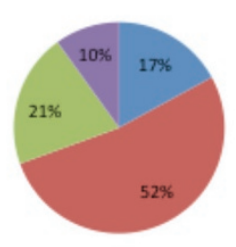

g

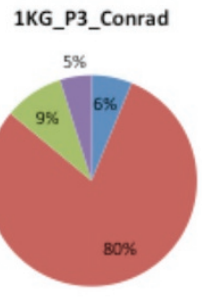

h

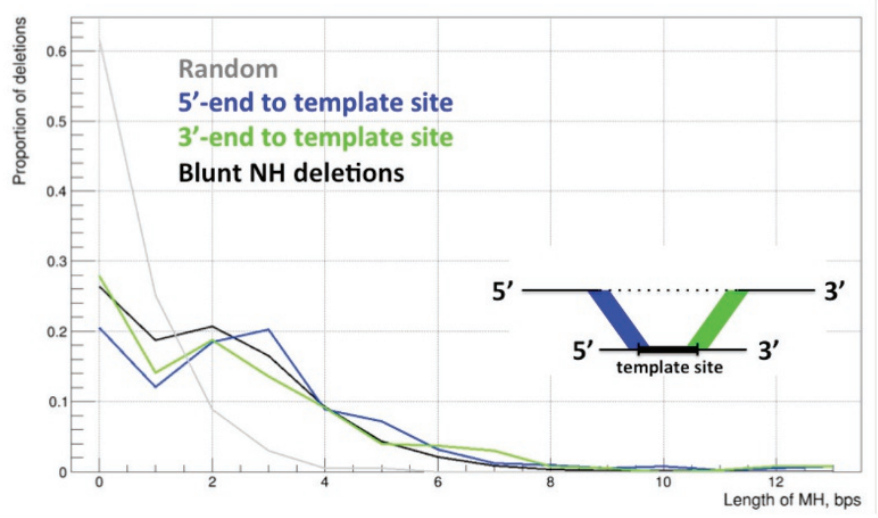

$=$ NAHR $=$ NHR $=$ TEI $=$ VNTR

Colored bases are direct copy and underlined are inverted sequences.

Ref Direct

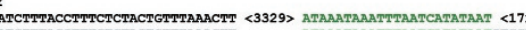

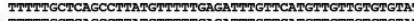

Left_Direct

7:120290250-120291387

ATPTGAATGTTGGCTTGCCTTGCTAGGTTGGGGAGTTCTCCTGGATAAT $<1137>$ CCTGGCTGCTGCCTTGCAGTTCGATCTCAGACTGCTGTGCCAGCAATGAG
ATTTGAATGTTGGCTTGCCTTGCTAGGTTGGGAAGTTCTCCTGGATAAT TCTCCTGGAATTCT OCTGGCTGCTGCCTTGCAGTTCGATCTCAGACTGCTGTGCCAGCAATGAG

Right Direct

GTGAAGGGATTTAAAAGCGGTTTGTGGCCTGCCATGGTGGCTCACCCCTA <79> AAACACCATCTCTACTAAAAATACAAAATCAGCCGGGTGTGGTGGTGGG

Ref_Inverted

CACAACCTCTGTCACTCCCGAGTCATCCTGCAAAACAAACAAACAAACA $<1400>$ TAAGCATGTGCAGGA
CACAACCTCTGTCACTCCCGAGTCATCCTGCANAAACAAACAAACAAACA

Left Inverted
$6: 169905557-169905650$

GCACCARTTCCATTPAAGCCTCAAAGCACCATCATAAGGTACATTTGTTAT <93> GCAGACTGATAGCATTCCPATACTCCAAGAATAGTCCTCAGACCAGAAAC

Right Inverted

Ref Direct\&Ref Inverted
$10: 132988684-1 \overline{3} 2991208$

AAGGCCTCCCTAAATCATTCTA 〈1329> GCTACTCGGGAGGCTGAGGCAGGAGAA <1168> CAAATTCACCAAGATATAATTCATCCATATAACAAAAGCCACTTGTACTC

Ref Direct\&Left Direct

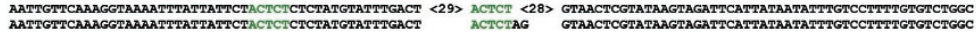


Extended Data Figure 9 $\mid$ SV clustering and breakpoint analysis. a-c, Extensive clustering of recurrent SVs into CNVRs appears unrelated to the extent of segmental duplications (a) and is only partially correlating with SNP diversity (b) and GC content (c). Breakdown of SV mechanism classifications based on criteria from two earlier studies (refs 6, 40). Shown are results for deletions with nucleotide resolved breakpoints. BreakSeq was used for mechanism inference. d, 1KG_P3: breakdown for our 1000 Genomes Project phase 3 SV callset using classification criteria from ref. 6. e, Conrad_2010: summary of mechanism classification results published in ref. 40.f, Mills_2011: summary of mechanism classification results published in ref. 6 .

g, 1KG_P3_Conrad: Breakdown for our 1000 Genomes Project phase 3 SV callset using classification criteria from ref. 40. Mechanism classification was pursued using four different categories. Blue, non-allelic homologous recombination (NAHR); green, mobile elements inserted into the reference genomes (appearing deleted in this analysis); red, non-homology-based rearrangement mechanisms (NHR), such as NHEJ, microhomology-mediated end-joining and microhomology-mediated break-induced replication (involving blunt-ended deletion breakpoints or breakpoints with microhomoloy); purple, expansion or shrinkage of variable numbers of tandem repeats (VNTRs). TEI, transposable element insertion (equivalent with MEI). h, Distribution of lengths of micro-homology $(\mathrm{MH})$ for complex SVs, measured between deletion and corresponding template sites boundaries. Simple deletions, which based on BreakSeq were inferred to be formed by a non-homology-based SV formation mechanism, such as NHEJ and microhomology-mediated break-induced replication (Supplementary Table 3), are shown as an additional control (here denoted 'blunt NH deletions'). i, Origins of inserted sequences in complex deletions inferred by split read analysis. This figure depicts examples for each class shown in Supplementary Table 13. 
ARTICLE RESEARCH

a

Class 1 - simple inv

d

Class 4 - inv-2dels
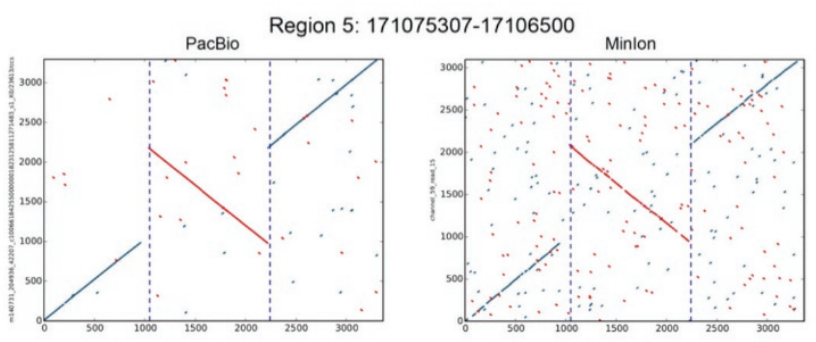

b

Class 2 - inv-dup
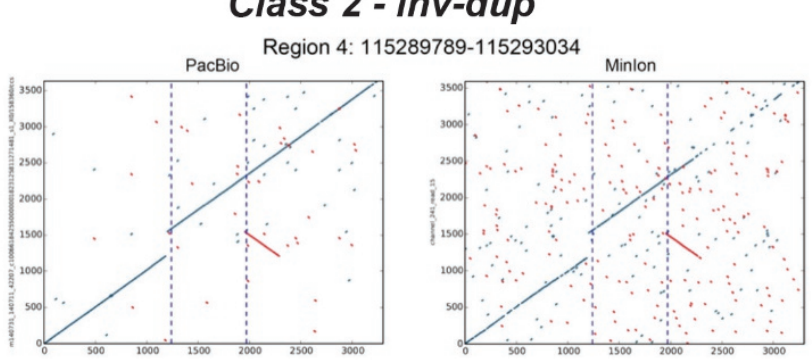

Class 3 - inv-del

C

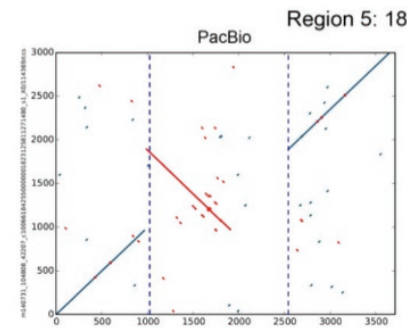

f
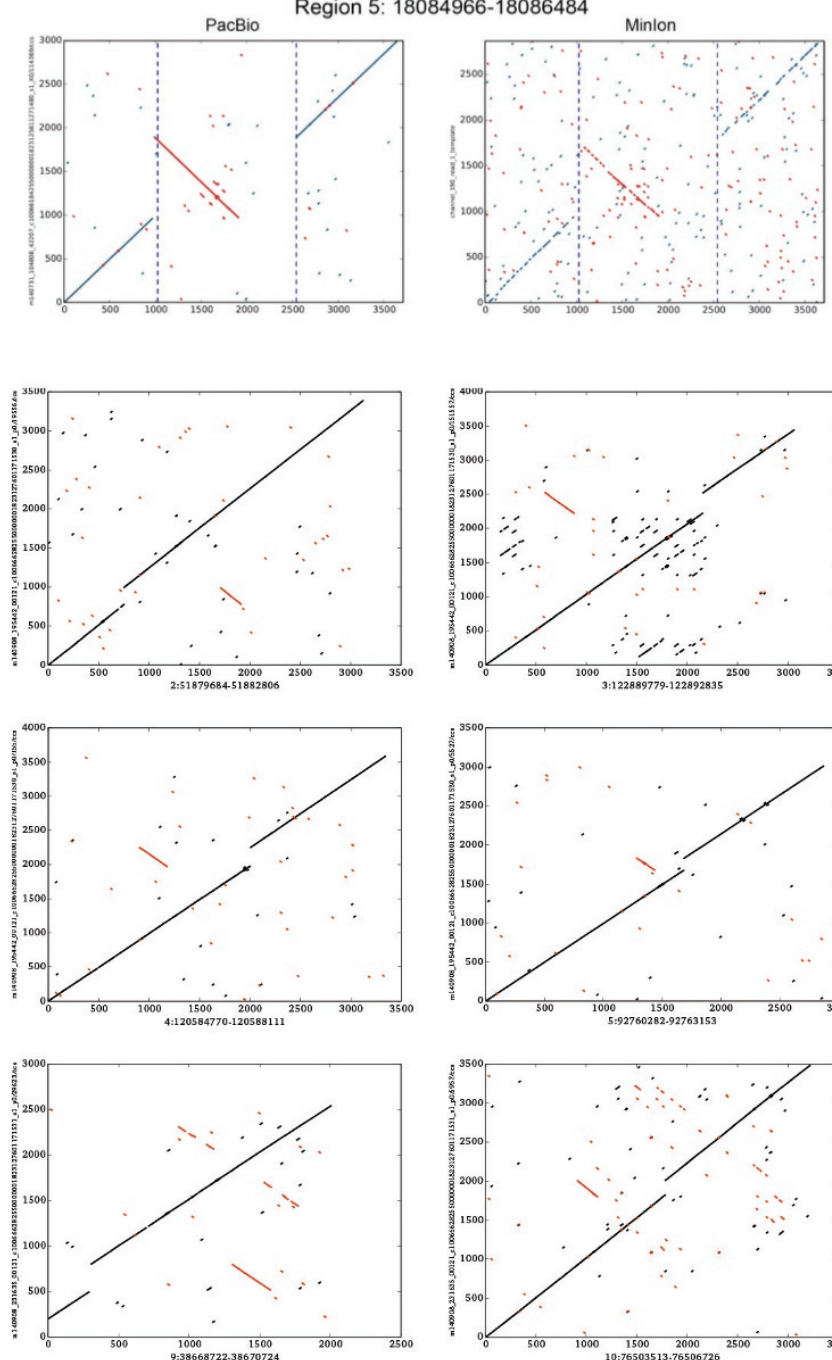
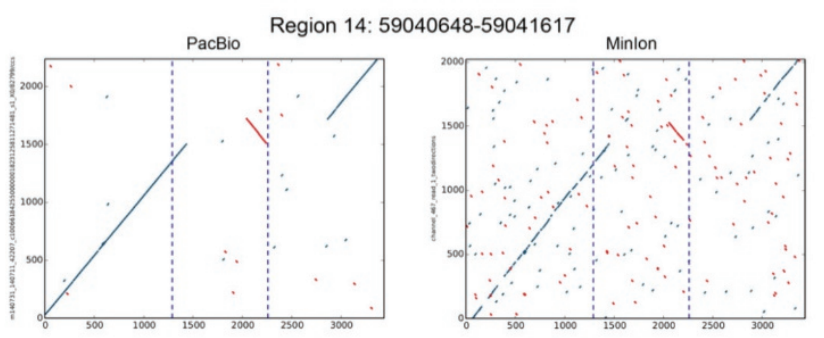

e

Class 5 - complex
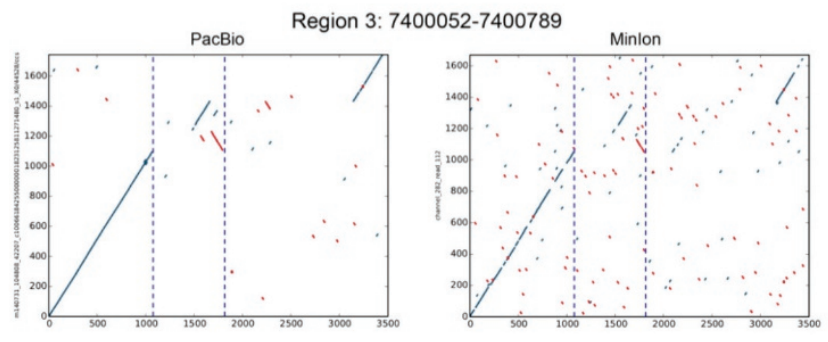
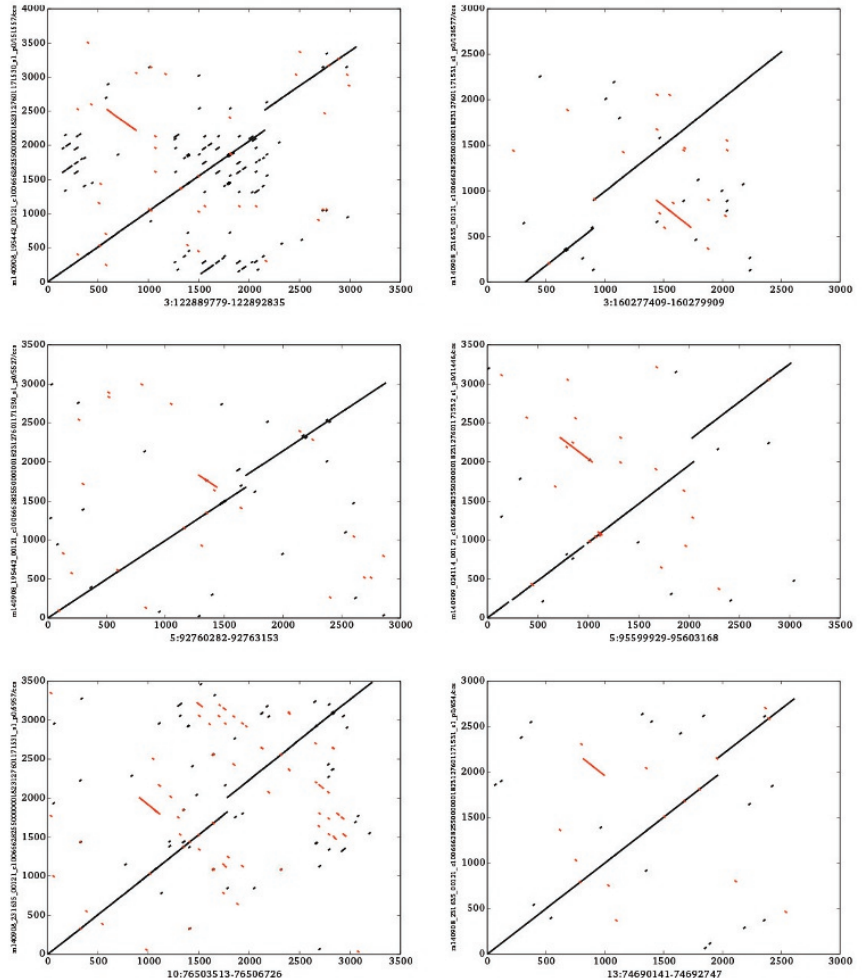
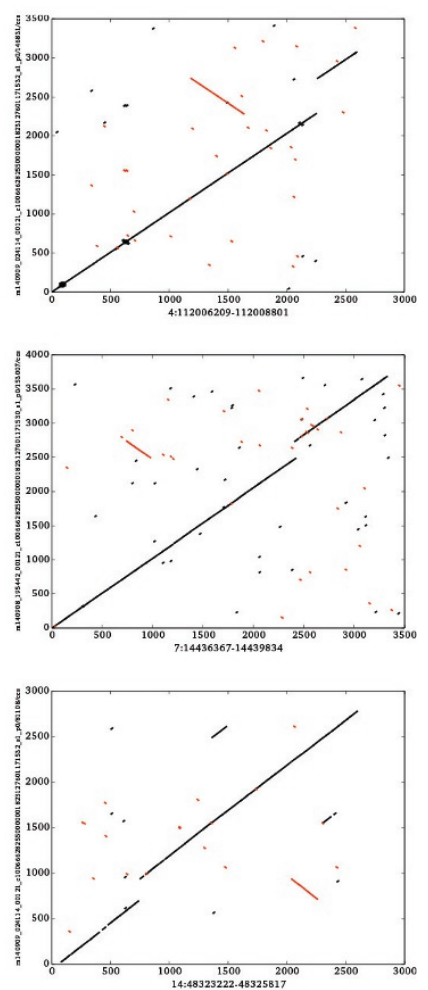

(C2015 Macmillan Publishers Limited. All rights reserved 


\section{RESEARCH ARTICLE}

Extended Data Figure 10 Examples of inversions identified in the SV release. a-e, Five classifications of inversions verified using PacBio and Minion reads are represented: Simple Inversion (a), inv-dup (b), inv-del (c), MultiDel with Inv (here abbreviated as inv-2dels) (d) and complex (e). f, Several further examples of inverted duplications (inv-dup), the most common form of inversion-associated SV identified in the phase 3 release set. The figure is depicting DNA sequence alignment dotplots (same arrangement as in Fig. 3), with the $y$ axis referring to PacBio DNA single molecule sequencing reads and the $x$ axis referring to the reference genome assembly (hg19). Inverted sequences are highlighted in red. Sequence analysis suggests that these inverted duplications are not typically associated with retrotransposition. 